Supplement of Atmos. Chem. Phys., 20, 9997-10014, 2020

https://doi.org/10.5194/acp-20-9997-2020-supplement

(c) Author(s) 2020. This work is distributed under

the Creative Commons Attribution 4.0 License.

(c) (i)
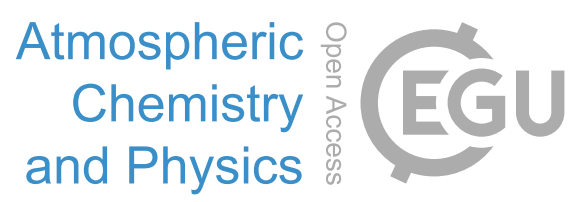

Supplement of

\title{
Polycyclic aromatic hydrocarbons (PAHs) and oxy- and nitro-PAHs in ambient air of the Arctic town Longyearbyen, Svalbard
}

Tatiana Drotikova et al.

Correspondence to: Tatiana Drotikova (tatiana.drotikova@unis.no)

The copyright of individual parts of the supplement might differ from the CC BY 4.0 License. 


\section{Table of Contents}

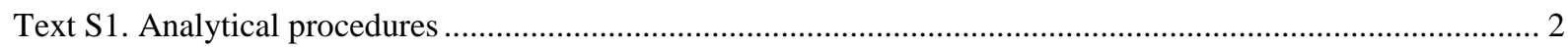

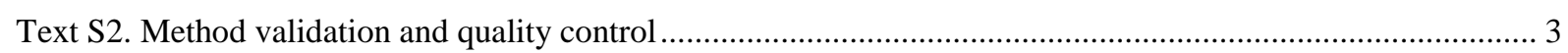

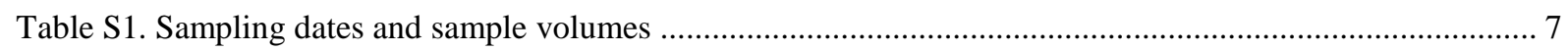

5 Table S2. Meteorological data for UNIS (samples U1-U7) and Adventdalen (samples A1-A7) sampling stations 7

Table S3. Physico-chemical properties of target compounds ......................................................................... 8

Table S4. GC-EI-MS/MS parameters used for PAHs determination and instrumental limits of quantification (LOQ).

Table S5. GC-ECNI-MS parameters used for nitro- and oxy-PAHs determination and instrumental limits of quantification (LOQ)

Table S6. Blank values and method detection limits (MDL) for different sampling materials......................... 16

Table S7. Average recovery rates (Rec, \%) and relative standard deviations (RSTD, \%) for spiked samples ..... 17

Table S8. Average recovery rates (Rec, \%) of internal standards (ISTDs) for ambient air samples .................. 19

Table S9. Concentrations of PAHs $(\mathrm{G}+\mathrm{P})$ and percentage in the particulate phase $(\% \mathrm{PM})$ in Longyearbyen power plant $(\mathrm{n}=6)$, as well as MDLs for gaseous (PUF) and particulate (QFF) phases, and instrumental LOD and LOQ; all values are in $\mathrm{pg} \mathrm{m}^{-3}$

Table S10. Concentrations of PAHs $(\mathrm{G}+\mathrm{P})$ and percentage in the particulate phase (\%PM) at $U N I S(\mathrm{n}=6)$, as well as MDLs for gaseous (PUF) and particulate (QFF) phases, and instrumental LOD and LOQ; all values are in pg $\mathrm{m}^{-3}$

20 Table S11. Concentrations of PAHs $(\mathrm{G}+\mathrm{P})$ and percentage in the particulate phase (\%PM) at Adventdalen ( $\mathrm{n}=6)$, as well as MDLs for gaseous (PUF) and particulate (QFF) phases, and instrumental LOD and LOQ; all values are in $\mathrm{pg} \mathrm{m}^{-3}$

Table S12. Comparison of Longyearbyen power plant $16 \mathrm{PAH}$ emissions with other coal-burning plants operated worldwide .....

25 Table S13. UNIS and Adventdalen air concentrations $(\mathrm{G}+\mathrm{P})$ of $16 \mathrm{PAHs}$ compared to national and regional background concentrations detected in autumn 2018

Table S14: Comparison of average concentrations $\left(\mathrm{G}+\mathrm{P} ; \mathrm{pg} \mathrm{m}^{-3}\right)$ of PAHs, oxy-PAHs and nitro-PAHs measured in Longyearbyen with those previously reported in the literature for rural sites worldwide.

Table S15. Spearman correlation of selected PAH \%PMs with ambient temperature and specific humidity for Adventdalen data $(n=6)$

Table S16. Spearman correlation of selected PAH concentrations $(\mathrm{G}+\mathrm{P})$ with precipitation for UNIS data ( $\mathrm{n}=6$ )

Table S17. Spearman correlation of PAH concentrations $(\mathrm{G}+\mathrm{P})$ with weather parameters for Adventdalen data

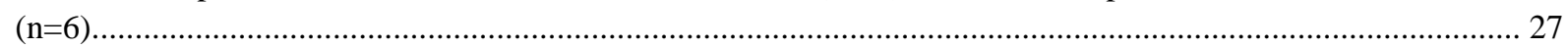

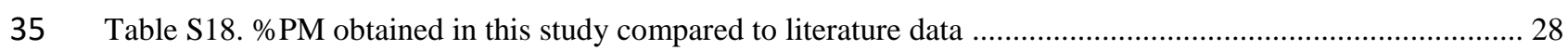

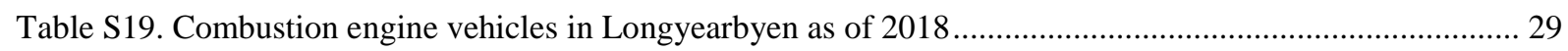

Table S20. Eigenanalysis of the correlation matrix and Eigenvectors for Adventdalen data............................ 30

Table S21. Spearman correlation of PAH concentrations $(\mathrm{G}+\mathrm{P} ; \mathrm{n}=6)$ with diagnostic ratios for Adventdalen data

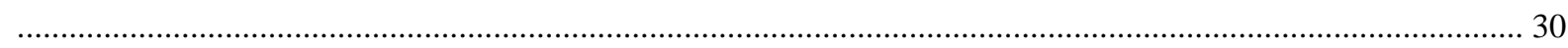

40 Table S22. Extractions from certificate of quality: unleaded gasoline, RON95, Norway, summer.................... 31

Table S23. Extractions from certificate of quality: B-base automotive diesel, CFPP-12, Norway ..................... 31

Table S24. Ratios of nitro- and oxy-PAH to corresponding parent PAH at three locations .............................. 32 
Table S25. Spearman correlation of nitro- and oxy-PAH to corresponding parent PAH ratios with weather parameters in Adventdalen $(n=6)$.

45 Table S26. Spearman correlation of concentrations (G+P) of PAHs, nitro- and oxy-PAHs with each other for

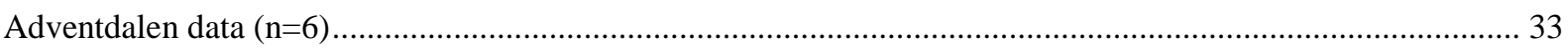

Table S27. Eigenanalysis of the correlation matrix and Eigenvectors for UNIS data....................................... 34

Table S28. Spearman correlation of PAH concentrations $(\mathrm{G}+\mathrm{P} ; \mathrm{n}=6)$ with diagnostic ratios for UNIS data ....... 34

Figure S1. Wind rose diagrams for UNIS (samples U1-U7) and Adventdalen (samples A1-A7) sampling stations

Figure S2. UNIS and Adventdalen chemical profiles of (a) PAHs, (b) oxy-PAHs, and (c) nitro-PAHs

Figure S3. The 15 PAH profiles for different stations in Svalbard (UNIS, Adventdalen, Zeppelin) and the mainland Norway (Birkenes) measured in autumn 2018 ........................................................................... 38

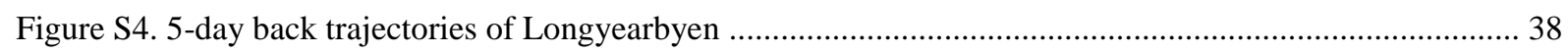

References

\section{Text S1. Analytical procedures}

\section{Chemicals}

Dichloromethane (DCM), $n$-hexane, and acetone (GC-MS grade), were purchased from VWR International AS,

Oslo, Norway. Standards of PAHs, nitro- and oxy-PAHs in $c$-hexane ( $>98.0 \%$ purity) were purchased from Chiron AS, Trondheim, Norway.

\section{Sample preparation}

QFFs (particulate phase) and PUFs (gaseous phase) samples were extracted separately by two different methods, followed by the same clean-up procedure. Fifty ng of $25{ }^{2} \mathrm{H}$-labelled PAH (dPAH) internal standards (ISTDs), including 16 EPA priority dPAHs (Table S4), 3 dOxy-PAHs, and 6 dNitro-PAHs (Table S5), were added before extraction. Previously reported methods (Albinet et al., 2006; Albinet et al., 2013; Albinet et al., 2014) were combined, modified and validated for the current trace quantitative analysis. QFF was placed in a centrifuge glass tube. After addition of 12-15 mL DCM, the tube was vortexed for $1.5 \mathrm{~min}$ (VWR 12620-848, Oslo, Norway). The sample was then centrifuged (Hettich, Universal 320, Germany) for 5 min at $4,000 \mathrm{rpm}$ at $10^{\circ} \mathrm{C}$ and the supernatant was transferred to a clean glass vial. The extraction procedure was repeated three times. Combined supernatants (about $40 \mathrm{ml}$ ) were concentrated to about $500 \mu \mathrm{L}$ under gentle nitrogen stream $(5.5$ quality; AGA, Norway) using Reacti-vap 18780 (Pierce Biotechnology Inc., Sweden). PUF samples were Soxhlet extracted with DCM for 24 hours. The extract (about $300 \mathrm{~mL}$ ) was reduced to about $500 \mu \mathrm{L}$ (Zymark, Turbovap 500, Sweden).

75 The QFF and PUF extracts were first cleaned on neutral alumina $\mathrm{Al}_{2} \mathrm{O}_{3}$ SPE cartridge (500 mg, Macherey Nagel, Germany). PAHs, nitro- and oxy-PAHs were eluted with $9 \mathrm{~mL}$ DCM. After concentration under a gentle nitrogen stream, the residue was dissolved in $200 \mu \mathrm{L} n$-hexane. Samples were further cleaned-up with neutral silica $\mathrm{SiO}_{2} \mathrm{SPE}$ cartridge (500 mg, Macherey Nagel, Germany). The alkane fraction was eluted with $1 \mathrm{~mL} n$ hexane and discarded. PAHs, nitro- and oxy-PAHs were thereafter eluted with $9 \mathrm{~mL}$ 35:65 (v/v) DCM- $n$-hexane.

80 The elute was dried under a gentle nitrogen stream and redissolved in $100 \mu \mathrm{L} n$-hexane. Subsequently, the purified samples were spiked with $10 \mathrm{ng}$ of three recovery standards (RSTDs; 1,2,3,4-tetrachloronaphthalene, Flt-d10, and 1-NPyr-d9) and analyzed by GC-MS. 


\section{GC-MS analysis}

16 priority PAHs, 8 oxy-PAHs, and 21 nitro-PAHs were analyzed via two different methods using a 7890B Agilent GC chromatograph coupled to 7000C Agilent Triple Quad MS (Agilent Technologies, Santa Clara, California). All compounds were separated on the low-polar TG-5SILMS capillary column (5\% Phenyl Methylpolysiloxane; $30 \mathrm{~m}$ with $5 \mathrm{~m}$ safe guard $\times 0.25 \mathrm{~mm} \times 0.25 \mu \mathrm{m}$ film thickness; cat. 26096-1425, Thermo Scientific Trace GC Ultra. A sample injection volume was $1 \mu \mathrm{L}$; pulsed splitless injection mode using a $4 \mathrm{~mm}$ ID splitless, single taper, no wool ultra inert liner (5190-2292, Agilent, USA). Agilent MassHunter software (Version B.07.00 /Build 7.0.457.0, 2008) was used for instrument control, method validation and quantification.

\section{PAH analysis by GC-EI-MS/MS method}

The injector temperature was $300{ }^{\circ} \mathrm{C}$ in pulsed splitless mode at $35 \mathrm{psi}$ for $1.5 \mathrm{~min}$ ( $1.6 \mathrm{~min}$ splitless time). Ultra pure He (quality 6.0; AGA, Norway) was used as carrier gas, at a constant flow rate of $1.0 \mathrm{~mL}$ min-1. The GC oven temperature program was as follows: initial temperature was hold at $70{ }^{\circ} \mathrm{C}$ for $3 \mathrm{~min}$, increased to $170{ }^{\circ} \mathrm{C}$ at $40{ }^{\circ} \mathrm{C}$ min-1, with further increase to $240{ }^{\circ} \mathrm{C}$ at $10{ }^{\circ} \mathrm{C}$ min-1, followed by a ramp to $310{ }^{\circ} \mathrm{C}$ at $5{ }^{\circ} \mathrm{C}$ min- 1 and hold for $2 \mathrm{~min}$. Transfer line temperature was $325^{\circ} \mathrm{C}$. The ion source temperature was $280{ }^{\circ} \mathrm{C}$ and quadrupoles temperatures were $150^{\circ} \mathrm{C}$.

The MS was run in electron ionization (EI) mode. The solvent delay time was $5.0 \mathrm{~min}$. Nitrogen (quality 6.0; AGA, Norway) was used as collision gas at a flow rate of $1.5 \mathrm{~mL}$ min-1. Helium quench gas was set at $2.25 \mathrm{~mL}$ min-1. Electron ionization was operated at $70 \mathrm{eV}$. Analyses were performed in multiple reaction monitoring (MRM) mode. Table S4 gives the retention times (RTs) and the monitored transitions for each compound and collision energy adopted from (Kanan et al., 2012). For the deuterated internal standards, the chosen transitions were parent molecular ion-parent molecular ion, at collision energy $0 \mathrm{eV}$.

\section{Nitro- and oxy-PAH analysis by GC-ECNI-MS method}

105 The injector temperature was $230^{\circ} \mathrm{C}$ in pulsed splitless mode at $40 \mathrm{psi}$ for $1.5 \mathrm{~min}$ ( $1.6 \mathrm{~min}$ splitless time). The carrier gas (He) flow rate was $1.2 \mathrm{~mL} \mathrm{~min}{ }^{-1}$. The $\mathrm{GC}$ temperature program started at $70{ }^{\circ} \mathrm{C}$ for $2 \mathrm{~min}$, then ramped to $250{ }^{\circ} \mathrm{C}$ at $45{ }^{\circ} \mathrm{C} \mathrm{min}^{-1}$ and held for $5 \mathrm{~min}$, followed by a ramp to $310{ }^{\circ} \mathrm{C}$ at $5{ }^{\circ} \mathrm{C} \mathrm{min}^{-1}$. Transfer line temperature was $325^{\circ} \mathrm{C}$.

The MS was run in electron capture negative ion (ECNI) mode. The MS parameters were as follows: ion source 110 temperature was $280{ }^{\circ} \mathrm{C}$ and quadrupole temperature was $150^{\circ} \mathrm{C}$. Methane (quality 6.0; AGA, Norway) was used as a reagent gas with a flow of $2.5 \mathrm{~mL} \mathrm{~min}^{-1}$, electron energy was $150 \mathrm{eV}$ and the emission current was 50 $\mu \mathrm{A}$. Analyses were performed in selected ion monitoring mode (SIM). Monitored ions and RT are shown in Table S5.

\section{Text S2. Method validation and quality control}

\section{Calibration}

Quantification of each individual PAH was based on eight-point calibration curve from 1 to $600 \mathrm{pg} \mu \mathrm{L}^{-1}$ (gravimetrically diluted) in $n$-hexane. Calibration curves were linear with $\mathrm{R}^{2}>0.987$ for all compounds. Quantification of nitro- and oxy-PAHs was based on nine-point calibration curves from 0.5 to $400 \mathrm{pg} \mu \mathrm{L}^{-1}$ (gravimetrically diluted) in $n$-hexane. Calibration curves were linear with $\mathrm{R}^{2}>0.99$ for all compounds. The

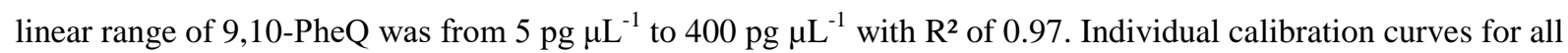


ISTDs were based on eight concentration levels $\left(5-250 \mathrm{pg} \mu \mathrm{L}^{-1}\right)$ with constant concentration of RSTDs (100 pg $\left.\mu \mathrm{L}^{-1}\right)$, prepared in $n$-hexane.

\section{Recovery rates}

Apparent recovery (Recovery, \%) for all analytes was calculated using Eq. (1):

125 Recovery, $\%=\frac{\mathrm{M}_{\mathrm{exp}}}{\mathrm{M}_{\mathrm{ref}}} \times 100$,

where $\mathbf{M}_{\text {exp }}$ is amount of target compound experimentally obtained from calibration graph and $\mathbf{M}_{\text {ref }}$ is a known added amount (Burns et al., 2002). Recovery of ISTD was calculated relative to RSTD added prior to GC analysis. Recoveries of target analytes were calculated relative to ISTD for spiked samples. The spiked samples were prepared by adding a known amount of native (16 PAHs, 19 nitro-PAHs, 8 oxy-PAHs) and internal (16 dPAHs, 6 dNitro-PAHs, 2 dOxy-PAHs) standards to the blank sample material before extraction. The spiked samples were treated as real samples. Four replicate samples for each sampling material were performed (QFF, $\mathrm{n}=4$ and PUF, $\mathrm{n}=4)$.

\section{Detection limits}

Instrumental limits of detection (LOD) and quantification (LOQ) were calculated according to the calibration curve method (Konieczka and Namieśnik, 2009; Shrivastava and Gupta, 2011; Şengül, 2016) based on residual standard deviation $\left(\mathrm{STDEV}_{\text {res }}\right)$ of the calibration curve in lowest concentration range (from 1 to 5 pg $\mu \mathrm{L}^{-1}$ for

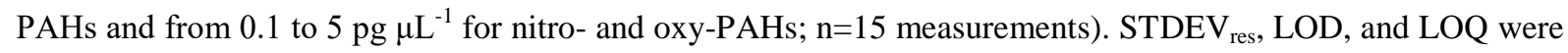
calculated by Eq. (2), (3) and (4), respectively.

STDEVres $=\sqrt{\frac{\sum(\mathrm{Y}-\mathrm{Yeq})^{2}}{\mathrm{n}-2}}$,

140 where $\mathrm{Y}$ is the observed value of a compound peak area and $\mathrm{Y}_{\mathrm{eq}}$ is the value calculated using the determined linear regression equation.

$\mathrm{LOD}=3.3 \times \mathrm{STDEV}_{\text {res }} /$ slope

$\mathrm{LOQ}=10 \times \mathrm{STDEV}_{\text {res }} /$ slope

Samples showed analyte concentrations below limit of quantification (LOQ) were replaced by LOQ/2 for statistical analysis.

In order to evaluate the background contamination related to sample collection and analysis, PUF and QFF field blanks (exposed filters without any air filtration; $n=4$ for PP and $n=4$ for UNIS/Adventdalen) and laboratory blanks ( $\mathrm{n}=3$ for PP and $\mathrm{n}=3$ for UNIS/Adventdalen) were treated and analyzed by the same methods as real samples. Laboratory blanks were prepared for each extraction batch. MDL was calculated based on blanks according to Eq. (5):

$\mathrm{MDL}=\overline{\mathrm{X}}+3 \times \mathrm{STD}_{\mathrm{b}}$

where $\overline{\mathrm{X}}$ is the blank mean concentration, and $\mathrm{STD}_{\mathrm{b}}$ is the standard deviation of the replicate blank sample concentrations. 


\section{Results of method validation and quality control}

Ambient atmospheric concentrations of nitro- and oxy-PAHs are in the range of few $\mathrm{pg} \mathrm{m}^{-3}$ to a few ng $\mathrm{m}^{-3}$, which are about 1-3 orders of magnitude lower than those of PAHs. Thus, a sensitive GC-ECNI-MS method was applied for the trace quantification of nitro- and oxy-PAHs. PAHs were identified based on compound specific RTs and two characteristic MRM transitions, while nitro- and oxy-PAHs were identified based on their RTs and monitored ions in SIM mode. The calculated instrumental LOD and LOQ values are summarized in Table S4 and Table S5. LOQ for PAHs (GC-EI-MS/MS) ranged from 0.98 to $3.69 \mathrm{pg}$. The HMW PAHs exhibited higher LODs. This may be due to interference from the stationary phase for later-eluting compounds. LOQ for nitro-PAHs (GC- ECNI-MS) ranged from 0.09 to $2.04 \mathrm{pg}$, while LOQ for oxy-PAHs (GC- ECNI-MS) were slightly higher, and ranged from 0.49 to $5.35 \mathrm{pg}$, and LOQ for 9,10-PheQ is $26.87 \mathrm{pg}$. This is because nitro-PAHs have higher affinity for negative ion formation, while the carbonyl group within oxy-PAHs is able to stabilize the excess negative charge associated with the capture of thermal electrons within the NICI process (Han et al., 2019). The linearity of instrumental response was evaluated over the range from 1 to $600 \mathrm{pg} \mu \mathrm{L}^{-1}$ for PAHs and 1 to $400 \mathrm{pg} \mu \mathrm{L}^{-1}$ for nitro- and oxy-PAHs. High values of regression coefficient $r^{2}$ were determined: $r^{2}$ $>0.987$ for all PAHs and $\mathrm{r}^{2}>0.990$ for all nitro- and oxy-PAHs, except 0.97 for 9,10-PheQ.

The recoveries percent of PAHs, nitro- and oxy-PAHs and their internal standards were calculated using spiked QFFs and PUFs samples. The recovery percent and accuracy (\%RSTD) results are summarized in Table S7. Relative standards deviations (RSTDs) were in the range 5-15\%, indicating a good repeatability. The recovery rates of native compounds from spiked QFFs samples were in the range 38-119\% for PAHs, 43-74 \% for NPAHs, and 38-57\% for OPAHs, while recoveries of labeled internal standards were $65-111 \%$ for dPAHs, 40$77 \%$ for dNitro-PAHs, and 40-41\% for dOxy-PAHs. The recovery rates of native compounds from spiked PUFs samples were in the range 44-121\% for PAHs, 56-104\% for nitro-PAHs, and 43-110\% for Oxy-PAHs, while recoveries of labeled internal standards were 54-101\% for dPAHs, 69-104\% for dNitro-PAHs, 50-74\% for dOxy-PAHs. Native nitro- and oxy-PAHs, such as BPyr-6, 6-NBaPyr, 1,3-, 1,6-, and 1,8-DNPyr, showed low recovery $(<30 \%)$ and therefore were excluded from quantification in air samples.

Samples spiking test (Table S7) showed that applying individual isotope labeled ISTD for each of 16 PAHs, resulted in higher apparent recovery rates ( $80 \%-100 \%$ for most of the compounds) compared to nitro- and oxyPAHs, where only 8 deuterated ISTDs were used for 31 nitro- and oxy-PAHs. Recovery rates for all ISTDs showed satisfying recoveries $(40-111 \%)$. dPAHs showed nearly equal, high extraction rates for the both methods applied for QFF and PUF spiked samples. The relatively low but still satisfying recovery for dNap, $54 \%$ for PUF and $65 \%$ for QFF, could be attributed to higher volatility of the compound, which leads to higher losses during sample preparation. dNitro- and dOxy-PAHs extracted from QFF exhibited lower recovery compared to those Soxhlet extracted from PUFs. This either indicates co-extraction of PUF matrix or higher extraction efficiency of nitro- and oxy-PAHs by hot solvent circulation through PUF over long period of time $(24 \mathrm{~h})$.

The ISTD recoveries obtained for QFF and PUF air samples (Table S8) were in acceptable range, 63-105\% for dPAHs, 56-69\% dOPAHs, 44-89\% dNAPHs.

Field $(n=8)$ and laboratory $(n=6)$ blanks were analysed in order to monitor and control possible contamination during sample transport and laboratory work. Method detection limit (Table S6) was determined based on blank levels. High contamination of blank samples by 9,10-PheQ (UNIS and Adventdalen), and 2-NFlu (PP) was 
found. Thus, concentrations for these compounds were excluded from the final results. No blank correction was 195 performed for the concentration calculations. 
Table S1. Sampling dates and sample volumes

\begin{tabular}{|c|c|c|c|c|c|c|c|c|}
\hline Sample & Date & $\begin{array}{c}\text { Power plant } \\
\text { Volume, } \mathrm{m}^{3}\end{array}$ & Sample & Start date* & $\begin{array}{r}\text { UNIS } \\
\text { Volume, } \mathrm{m}^{3} \\
\end{array}$ & Sample & Start date* & $\begin{array}{l}\text { Adventdalen } \\
\text { Volume, } \mathrm{m}^{3}\end{array}$ \\
\hline PP1 & 27.09 .2018 & 2.7 & U1 & 28.08 .2018 & 349.2 & A1 & 28.08 .2018 & 359.5 \\
\hline PP2 & 27.09 .2018 & 3.0 & $\mathrm{U} 2$ & 30.08 .2018 & 376.1 & A2 & 30.08 .2018 & 349.7 \\
\hline PP3 & 27.09 .2018 & 1.7 & U4 & 13.09 .2018 & 365.6 & A3 & 06.09 .2018 & 451.5 \\
\hline PP4 & 02.10 .2018 & 1.5 & U5 & 25.09 .2018 & 384.5 & A4 & 13.09.2018 & 354.2 \\
\hline PP5 & 02.10 .2018 & 1.3 & U6 & 26.09 .2018 & 355.7 & A5 & 25.09 .2018 & 403.8 \\
\hline PP6 & 02.10 .2018 & 1.5 & U7 & 27.09 .2018 & 365.2 & A7 & 27.09 .2018 & 272.1 \\
\hline
\end{tabular}

*sampling duration 23-31 hours

Table S2. Meteorological data for UNIS (samples U1-U7) and Adventdalen (samples A1-A7) sampling stations

\begin{tabular}{|c|c|c|c|c|c|c|c|c|}
\hline $\begin{array}{l}\text { Sample } \\
\text { name }\end{array}$ & 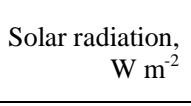 & $\begin{array}{l}\text { Air temp, } \\
{ }^{\circ} \mathrm{C}\end{array}$ & $\begin{array}{r}\text { Pressure, } \\
\mathrm{hPa}\end{array}$ & $\begin{array}{r}\mathrm{RH}, \\
\%\end{array}$ & $\begin{array}{r}\text { Specific } \\
\text { humidity*, } \\
\mathrm{kg} \mathrm{kg}^{-1}\end{array}$ & $\begin{array}{l}\text { Wind from }{ }^{* *}, \\
\text { degree }\end{array}$ & $\begin{array}{r}\text { Precipitation, } \\
\mathrm{mm}\end{array}$ & $\begin{array}{r}\text { Type of } \\
\text { precipitation }\end{array}$ \\
\hline U1 & $88.7 \pm 137.6$ & $5.2 \pm 1.0$ & 1011.5 & 74.7 & 0.0041 & 130.0 & 0.1 & rain \\
\hline $\mathrm{U} 2$ & $42.2 \pm 45.3$ & $4.7 \pm 0.9$ & 1000.1 & 78.0 & 0.0042 & 230.0 & 4.2 & rain \\
\hline U4 & $31.0 \pm 34.7$ & $1.2 \pm 0.9$ & 1003.3 & 79.5 & 0.0033 & 150.0 & 0.0 & - \\
\hline U5 & $18.3 \pm 24.4$ & $-1.1 \pm 1.3$ & 984.3 & 83.7 & 0.0030 & 220.0 & 2.8 & snow \\
\hline U6 & $27.9 \pm 39.0$ & $-3.3 \pm 0.5$ & 990.4 & 67.5 & 0.0020 & 260.0 & 0.3 & snow \\
\hline U7 & $16.9 \pm 28.7$ & $-3.1 \pm 0.4$ & 994.2 & 68.9 & 0.0020 & 260.0 & 0.2 & snow \\
\hline A1 & $88.7 \pm 137.6$ & $4.9 \pm 0.9$ & 1011.5 & 77.0 & 0.0041 & 120.0 & 0.1 & rain \\
\hline $\mathrm{A} 2$ & $42.2 \pm 45.3$ & $5.0 \pm 1.0$ & 1000.1 & 75.4 & 0.0042 & 260.0 & 4.2 & rain \\
\hline $\mathrm{A} 3$ & $76.4 \pm 110.7$ & $3.1 \pm 1.0$ & 1016.9 & 78.9 & 0.0040 & 270.0 & 0.0 & - \\
\hline A4 & $31.0 \pm 34.7$ & $0.7 \pm 0.8$ & 1003.3 & 85.4 & 0.0033 & 120.0 & 0.0 & - \\
\hline A5 & $18.3 \pm 24.4$ & $-1.8 \pm 1.0$ & 984.3 & 82.2 & 0.0030 & 210.0 & 2.8 & snow \\
\hline A7 & $16.9 \pm 28.7$ & $-2.7 \pm 0.3$ & 994.2 & 63.5 & 0.0020 & 250.0 & 0.2 & snow \\
\hline
\end{tabular}

*calculated according to Launiainen and Vihma (1990)

***wind roses are presented as Figure S1 
Table S3. Physico-chemical properties of target compounds

\begin{tabular}{|c|c|c|c|c|c|c|c|c|}
\hline Compound & Abbrevation & CAS number & Structure $^{1)}$ & $\log \mathrm{K}_{\mathrm{oa}}^{2)}$ & $\begin{array}{r}\text { Water solubility, } \\
{\text { (estimated })^{2)}}^{m g ~ L^{-1}} \\
\end{array}$ & $\begin{array}{r}\text { Water solubility } \\
(\text { experimental) } \\
\mathrm{mg} \mathrm{L}^{-1}\end{array}$ & $\begin{array}{r}\text { Boiling point }{ }^{3)} \\
{ }^{\circ} \mathrm{C}\end{array}$ & $\log \mathrm{K}_{\mathrm{ow}}{ }^{2)}$ \\
\hline Naphthalene & Nap & $91-20-3$ & & 5.04 & 142.1 & 31.0 & $221.5 \pm 7.0$ & 3.30 \\
\hline Acenaphthylene & Acy & $208-96-8$ & & 6.27 & 2.49 & 16.1 & $298.9 \pm 7.0$ & 3.94 \\
\hline Acenaphthene & Ace & $83-32-9$ & & 6.04 & 2.53 & 3.90 & $279.0 \pm 0.0$ & 3.92 \\
\hline Fluorene & Flu & $86-73-7$ & & 6.59 & 1.34 & 1.69 & $293.6 \pm 10.0$ & 4.18 \\
\hline Phenanthrene & Phe & $85-01-8$ & & 7.22 & 0.68 & 1.15 & $337.4 \pm 9.0$ & 4.46 \\
\hline Anthracene & Ant & $120-12-7$ & & 7.09 & 0.69 & 0.04 & $337.4 \pm 9.0$ & 4.45 \\
\hline Fluoranthene & Flt & $206-44-0$ & & 8.60 & 0.13 & 0.26 & $375.0 \pm 0.0$ & 5.16 \\
\hline Pyrene & Pyr & $129-00-0$ & & 8.19 & 0.22 & 0.14 & $404.0 \pm 0.0$ & 4.88 \\
\hline Benzo(a)anthracene & BaAnt & $56-55-3$ & & 9.07 & 0.03 & 0.009 & $436.7 \pm 12.0$ & 5.76 \\
\hline Chrysene & Chry & 218-01-9 & & 9.48 & 0.03 & 0.002 & $448.0 \pm 0.0$ & 5.81 \\
\hline
\end{tabular}




\begin{tabular}{|c|c|c|c|c|c|c|c|}
\hline Benzo(b)fluoranthene & BbFlt & $205-99-2$ & 10.35 & 0.02 & 0.002 & $467.5 \pm 12.0$ & 5.78 \\
\hline Benzo(k)fluoranthene & BaFlt & $206-44-0$ & 8.60 & 0.13 & 0.26 & N.A. & 5.16 \\
\hline Benzo(a)pyrene & $\mathrm{BaPyr}$ & $50-32-8$ & 10.86 & 0.01 & 0.002 & $495.0 \pm 0.0$ & 6.13 \\
\hline Indeno(1,2,3-cd)pyrene & IPyr & $193-39-5$ & 11.55 & 0.002 & 0.0002 & $497.1 \pm 12.0$ & 6.70 \\
\hline Dibenzo(ah)anthracene & DBAnt & $53-70-3$ & 11.78 & 0.003 & 0.001 & $524.7 \pm 17.0$ & 6.54 \\
\hline Benzo(g,h,i)perylene & BPer & $191-24-2$ & 11.50 & 0.003 & 0.0003 & $501.0 \pm 0.0$ & 6.63 \\
\hline 1,4-Naphthoquinone & 1,4-NapQ & $130-15-4$ & 8.80 & 2417 & N.A. & $297.9 \pm 40.0$ & 1.71 \\
\hline 1-Nitronaphthalene & 1-NNap & $86-57-7$ & 7.33 & 45.66 & 9.18 & $304.0 \pm 0.0$ & 3.19 \\
\hline 2-Nitronaphthalene & 2-NNap & $581-89-5$ & 7.31 & 41.38 & 9.24 & $319.6 \pm 11.0$ & 3.24 \\
\hline
\end{tabular}




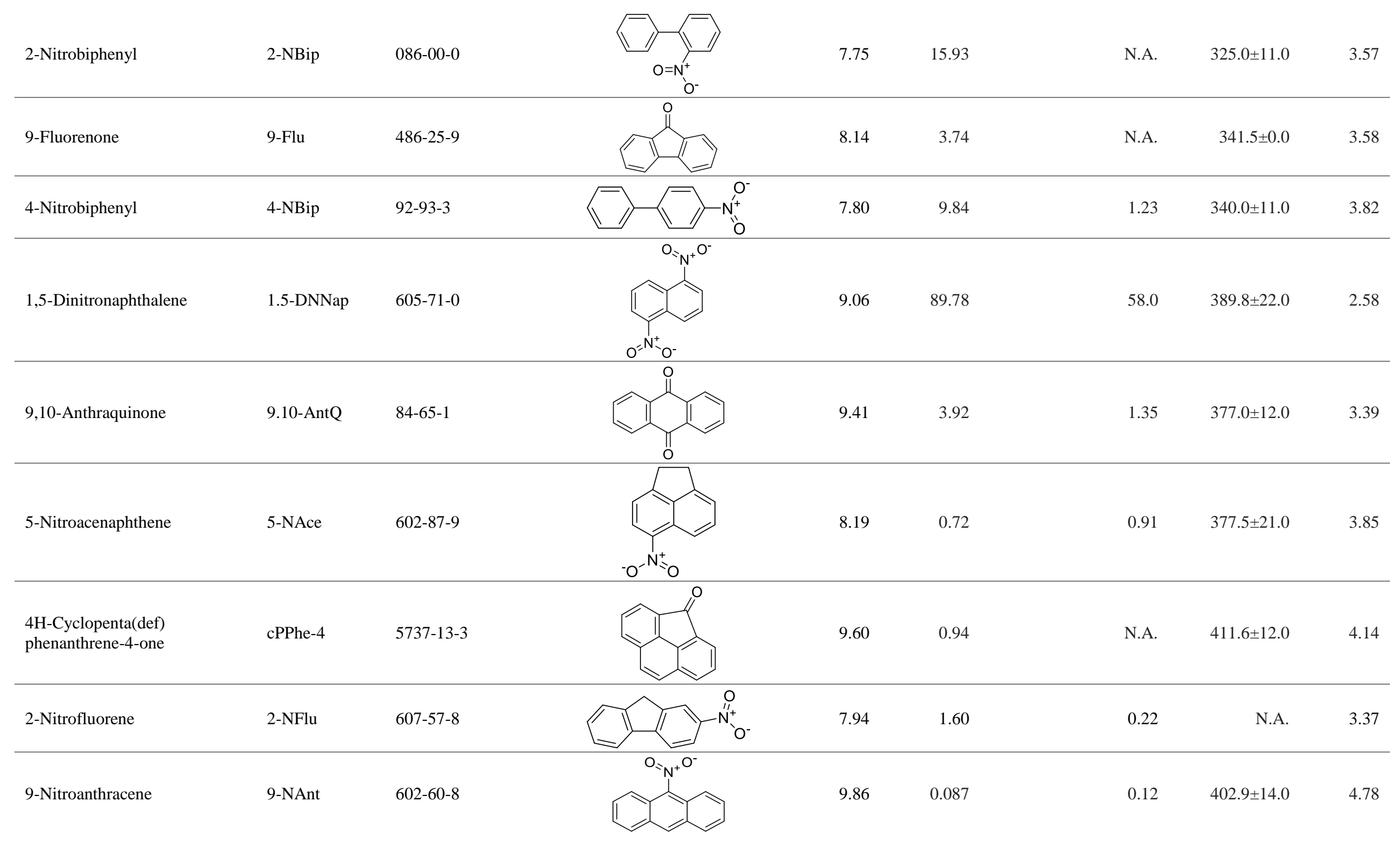




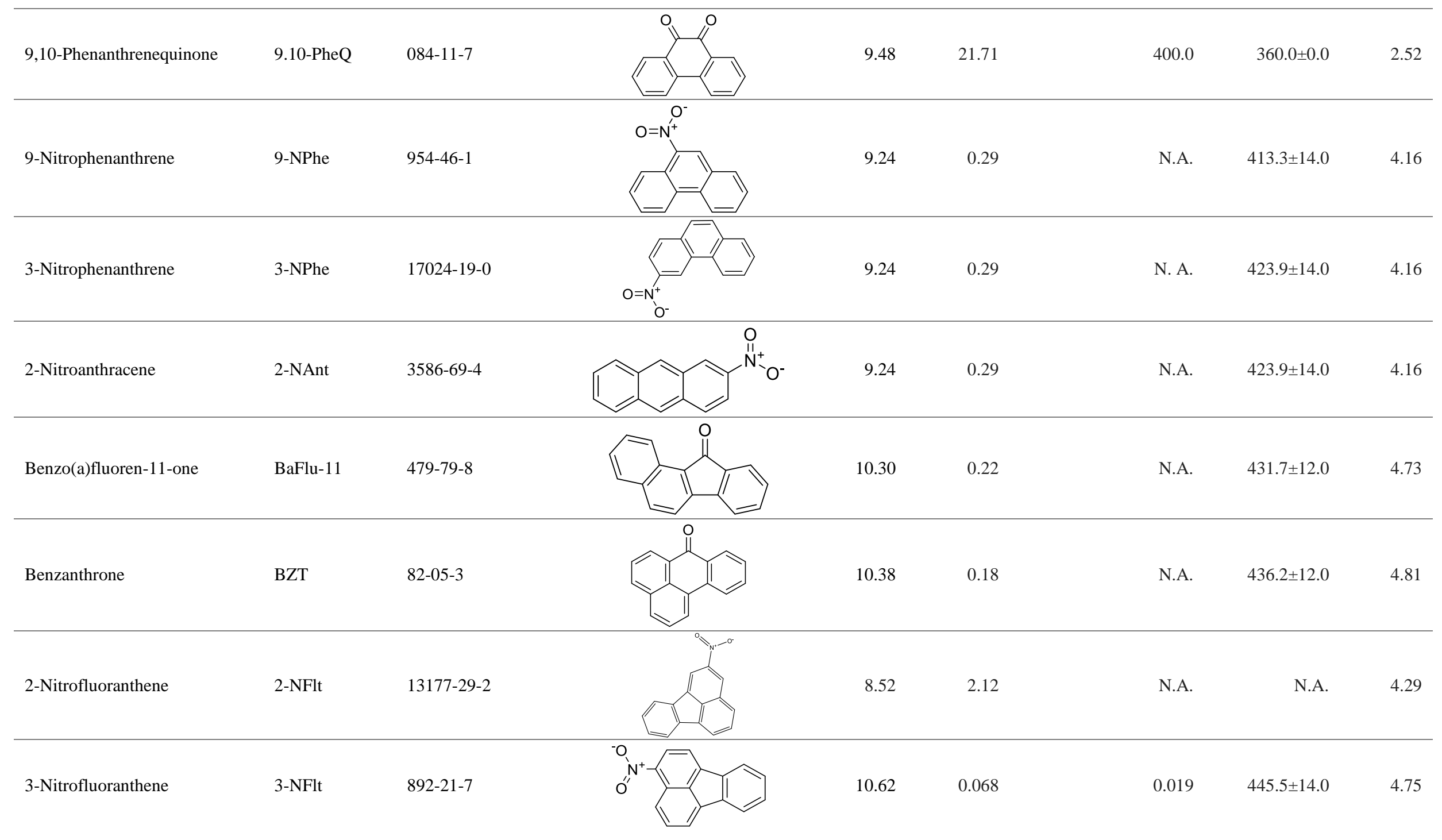




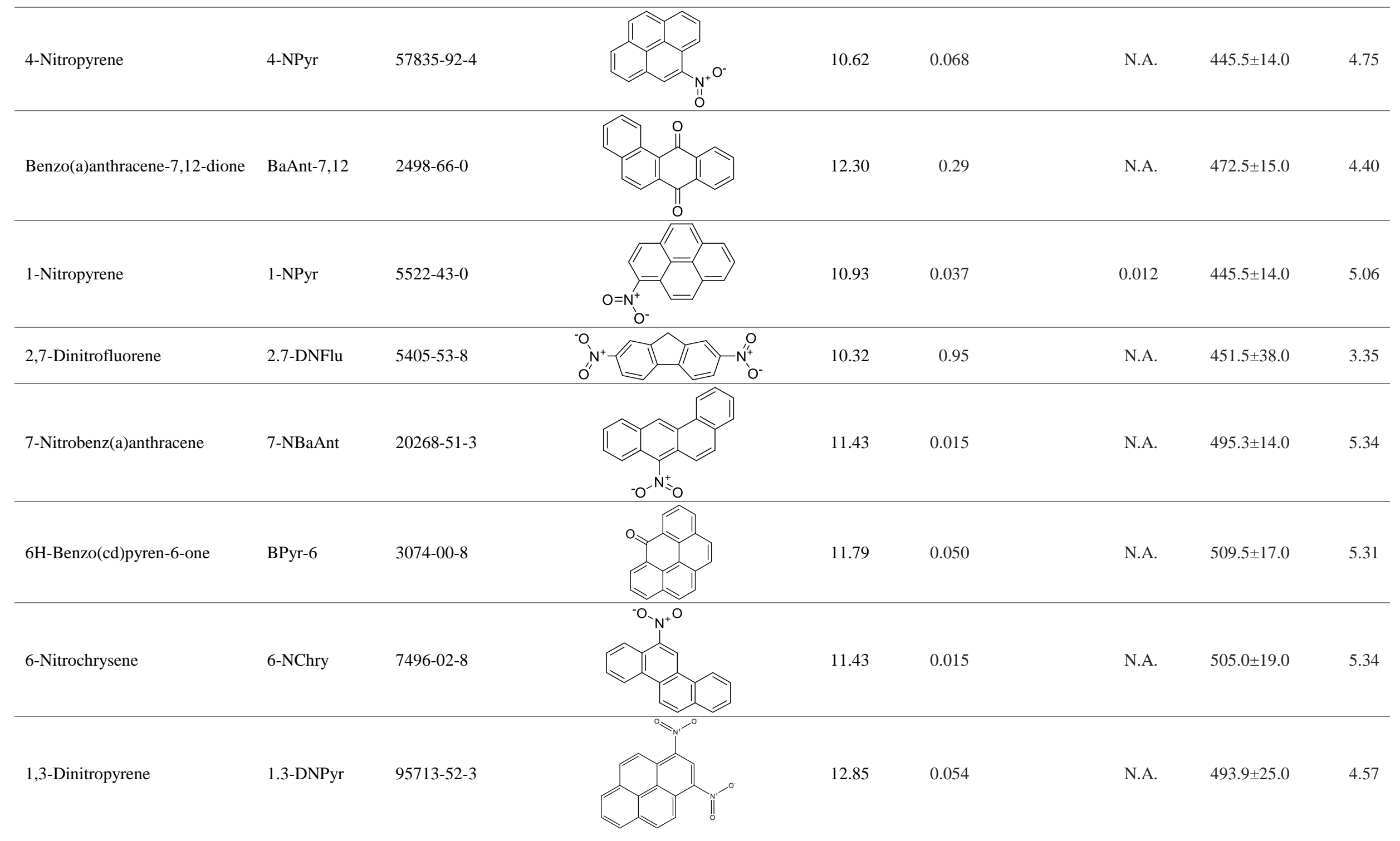




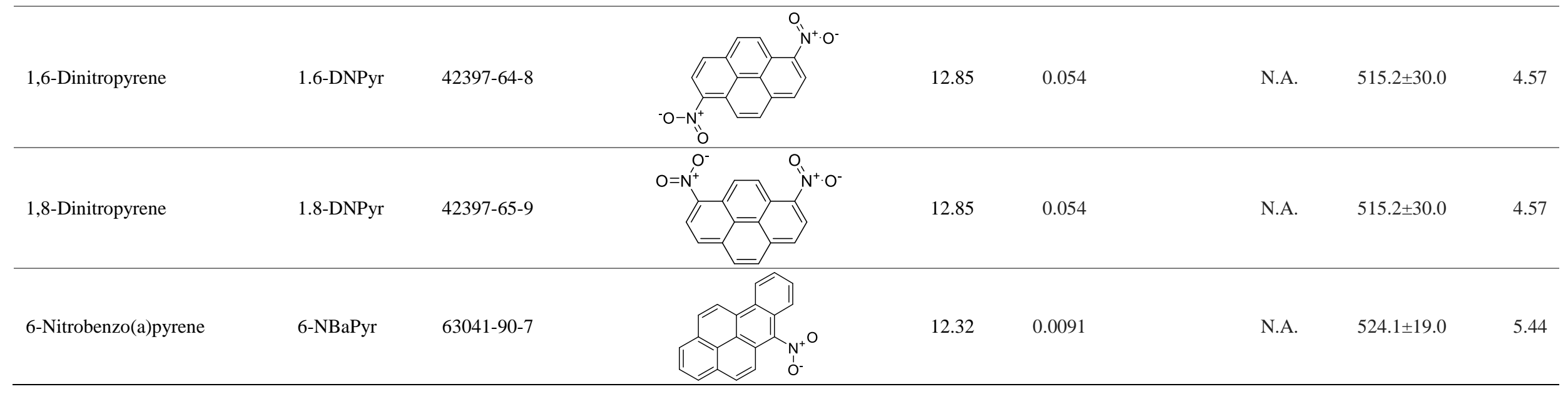

1) All structures were prepared with ChemDraw Professional, v 15.0.0.106, PerkinElmer Informatics, Inc., Boston, Massachusetts, USA, 2015

${ }^{2)}$ Acquired from EPI Suite, U.S.EPA: Estimation Programs Interface Suite, v 4.11, United States Environmental Protection Agency, Washington, DC, USA, 2019

${ }^{3)}$ Predicted data are calculated with ACD/Labs Percepta Platform - PhysChem Module, Toronto, Canada, 2015 
Table S4. GC-EI-MS/MS parameters used for PAHs determination and instrumental limits of quantification (LOQ)

\begin{tabular}{|c|c|c|c|c|c|c|}
\hline Compound & $\begin{array}{r}\text { Retention time, } \\
\min \end{array}$ & $\begin{array}{r}\text { Precursor ion, } \\
\mathrm{m} / \mathrm{z}\end{array}$ & $\begin{array}{l}\text { Product ion } \\
\text { quantifier, } \\
\mathrm{m} / \mathrm{z}\end{array}$ & $\begin{array}{r}\text { Product ion } \\
\text { qualifier, } \\
\mathrm{m} / \mathrm{z}\end{array}$ & $\begin{array}{r}\text { Collision energy, } \\
\mathrm{eV}\end{array}$ & $\begin{array}{r}\text { LOQ, } \\
\text { pg }\end{array}$ \\
\hline Naphthalene-d8 & 6.20 & 136 & 136 & - & $\mathbf{0}$ & - \\
\hline Naphthalene & 6.22 & 128 & 102 & 127 & $20 / 20$ & 1.74 \\
\hline Acenaphtylene-d8 & 7.88 & 160 & 160 & - & $\mathbf{0}$ & - \\
\hline Acenaphtylene & 7.89 & 152 & 151 & 150 & $25 / 25$ & 1.29 \\
\hline Acenaphthene-d10 & 8.07 & 164 & 164 & - & $\mathbf{0}$ & - \\
\hline Acenaphthene & 8.11 & 154 & 152 & 153 & $35 / 35$ & 1.27 \\
\hline Fluorene-d10 & 8.81 & 176 & 176 & - & $\mathbf{0}$ & - \\
\hline Fluorene & 8.86 & 166 & 165 & 164 & $40 / 40$ & 1.14 \\
\hline Phenanthrene-d10 & 10.46 & 188 & 188 & - & $\mathbf{0}$ & - \\
\hline Phenanthrene & 10.50 & 178 & 176 & 152 & $40 / 15$ & 1.43 \\
\hline Anthracene-d10 & 10.56 & 188 & 188 & - & $\mathbf{0}$ & - \\
\hline Anthracene & 10.56 & 178 & 176 & 152 & $40 / 15$ & 1.56 \\
\hline Fluoranthnene-d10 & 12.91 & 212 & 212 & - & $\mathbf{0}$ & - \\
\hline Fluoranthnene & 12.95 & 202 & 201 & 200 & $20 / 20$ & 1.32 \\
\hline Pyrene-d10 & 13.43 & 212 & 212 & - & $\mathbf{0}$ & - \\
\hline Pyrene & 13.47 & 202 & 201 & 200 & $20 / 20$ & 0.98 \\
\hline Benzo(a)anthracene-d12 & 16.78 & 240 & 240 & - & $\mathbf{0}$ & - \\
\hline Benzo(a)anthracene & 16.84 & 228 & 226 & 227 & $30 / 30$ & 3.39 \\
\hline Chrysene-d12 & 16.86 & 240 & 240 & - & $\mathbf{0}$ & - \\
\hline Chrysene & 16.94 & 228 & 226 & 227 & $30 / 30$ & 2.92 \\
\hline Benzo(b+k)fluoranthene-d12 & 20.45 & 264 & 264 & - & $\mathbf{0}$ & - \\
\hline Benzo $(b+k)$ fluoranthene & 20.50 & 252 & 250 & 251 & $25 / 25$ & 2.88 \\
\hline Benzo(a)pyrene-d12 & 21.45 & 264 & 264 & - & $\mathbf{0}$ & - \\
\hline Benzo(a)pyrene & 21.52 & 252 & 250 & 251 & $25 / 25$ & 2.95 \\
\hline Indeno(1,2,3-cd)pyrene-d12 & 25.14 & 288 & 288 & - & $\mathbf{0}$ & - \\
\hline Indeno(1,2,3-cd)pyrene & 25.22 & 276 & 274 & 275 & $35 / 35$ & 3.69 \\
\hline $\operatorname{Dibenzo}(\mathbf{a}, \mathbf{h})$ anthracene-d12 & 25.21 & 292 & 292 & - & $\mathbf{0}$ & - \\
\hline Dibenzo(a,h)anthracene & 25.30 & 278 & 276 & 277 & $25 / 25$ & 3.54 \\
\hline Benzo(g,h,i)perylene-d14 & 25.91 & 288 & 288 & - & $\mathbf{0}$ & - \\
\hline Benzo(g,h,i)perylene & 25.98 & 276 & 274 & 275 & $35 / 35$ & 3.37 \\
\hline
\end{tabular}


Table S5. GC-ECNI-MS parameters used for nitro- and oxy-PAHs determination and instrumental limits of quantification (LOQ)

\begin{tabular}{|c|c|c|c|}
\hline Compound & Monitored ion, $\mathrm{m} / \mathrm{z}$ & Retention time, min & LOQ, pg \\
\hline 1,4-Naphthaquinone-d6 & 164 & 5.95 & - \\
\hline 1,4-Naphthaquinone & 158 & 5.96 & 0.12 \\
\hline 1-Nitronaphthalene-d7 & 180 & 6.50 & - \\
\hline 1-Nitronaphthalene & 173 & 6.60 & 0.09 \\
\hline 2-Nitronaphthalene & 173 & 6.90 & 0.09 \\
\hline 2-Nitrobiphenyl-d9 & 208 & 6.76 & - \\
\hline 2-Nitrobiphenyl & 199 & 6.80 & 0.13 \\
\hline 9-Fluorenone-d8 & 188 & 6.90 & - \\
\hline 9-Fluorenone & 180 & 7.00 & 0.49 \\
\hline 4-Nitrobiphenyl & 199 & 7.35 & 0.24 \\
\hline 1,5-Dinitronaphtalene & 218 & 7.60 & 0.23 \\
\hline Anthraquinone-d8 & 216 & 7.80 & - \\
\hline 9,10-Anthraquinone & 208 & 7.85 & 0.84 \\
\hline 1,2,3,4-Tetrachloronaphthalene RSTD & 264 & 7.80 & - \\
\hline 5-Nitroacenaphthene & 199 & 8.00 & 0.20 \\
\hline 4H-Cyclopenta[def]phenanthrene-4-one & 204 & 8.20 & 0.50 \\
\hline Fluoranthene-d10 RSTD & 212 & 8.30 & - \\
\hline 2-Nitrofluorene & 211 & 8.50 & 0.26 \\
\hline 2-Nitrofluorene-d9 & 220 & 8.50 & - \\
\hline 9-Nitroanthracene-d9 & 232 & 8.66 & - \\
\hline 9-Nitroanthracene & 223 & 8.70 & 0.33 \\
\hline 9,10-Phenanthrenequinone & 208 & 8.95 & 26.87 \\
\hline 9-Nitrophenanthrene & 223 & 9.20 & 0.37 \\
\hline 3-Nitrophenantherene & 223 & 9.50 & 0.57 \\
\hline 2-Nitroanthracene & 223 & 9.90 & 0.58 \\
\hline Benzo[a]fluoren-11-one & 230 & 10.40 & 0.75 \\
\hline Benzanthrone & 230 & 11.90 & 2.69 \\
\hline 3-Nitrofluoranthene-d9 & 256 & 12.40 & - \\
\hline 2-Nitrofluoranthene & 247 & 12.40 & 1.36 \\
\hline 3-Nitrofluoranthene & 247 & 12.50 & 1.22 \\
\hline 4-Nitropyrene & 247 & 12.70 & 1.12 \\
\hline Benzo[a]anthracene-7,12-dione & 258 & 13.00 & 1.62 \\
\hline 1-Nitropyrene-d9 RSTD & 256 & 13.20 & - \\
\hline 1-Nitropyrene & 247 & 13.20 & 1.21 \\
\hline 2-Nitropyrene & 247 & 13.20 & 2.30 \\
\hline 2,7-Dinitrofluorene & 256 & 13.80 & 1.41 \\
\hline 7-Nitrobenzo[a]anthracene & 273 & 15.70 & 1.22 \\
\hline 6H-Benzo[cd]pyren-6-one & 254 & 16.50 & 5.35 \\
\hline 6-Nitrochrysene-d11 & 284 & 16.80 & - \\
\hline 6-Nitrochrysene & 273 & 16.90 & 1.30 \\
\hline 1,3-Dinitropyrene & 292 & 17.80 & 1.27 \\
\hline 1,6-Dinitropyrene & 292 & 18.60 & 2.04 \\
\hline 1,8-Dinitropyrene & 292 & 19.10 & 1.55 \\
\hline 6-Nitrobenzo(a)pyrene & 297 & 21.00 & 1.14 \\
\hline
\end{tabular}


Table S6. Blank values and method detection limits (MDL) for different sampling materials

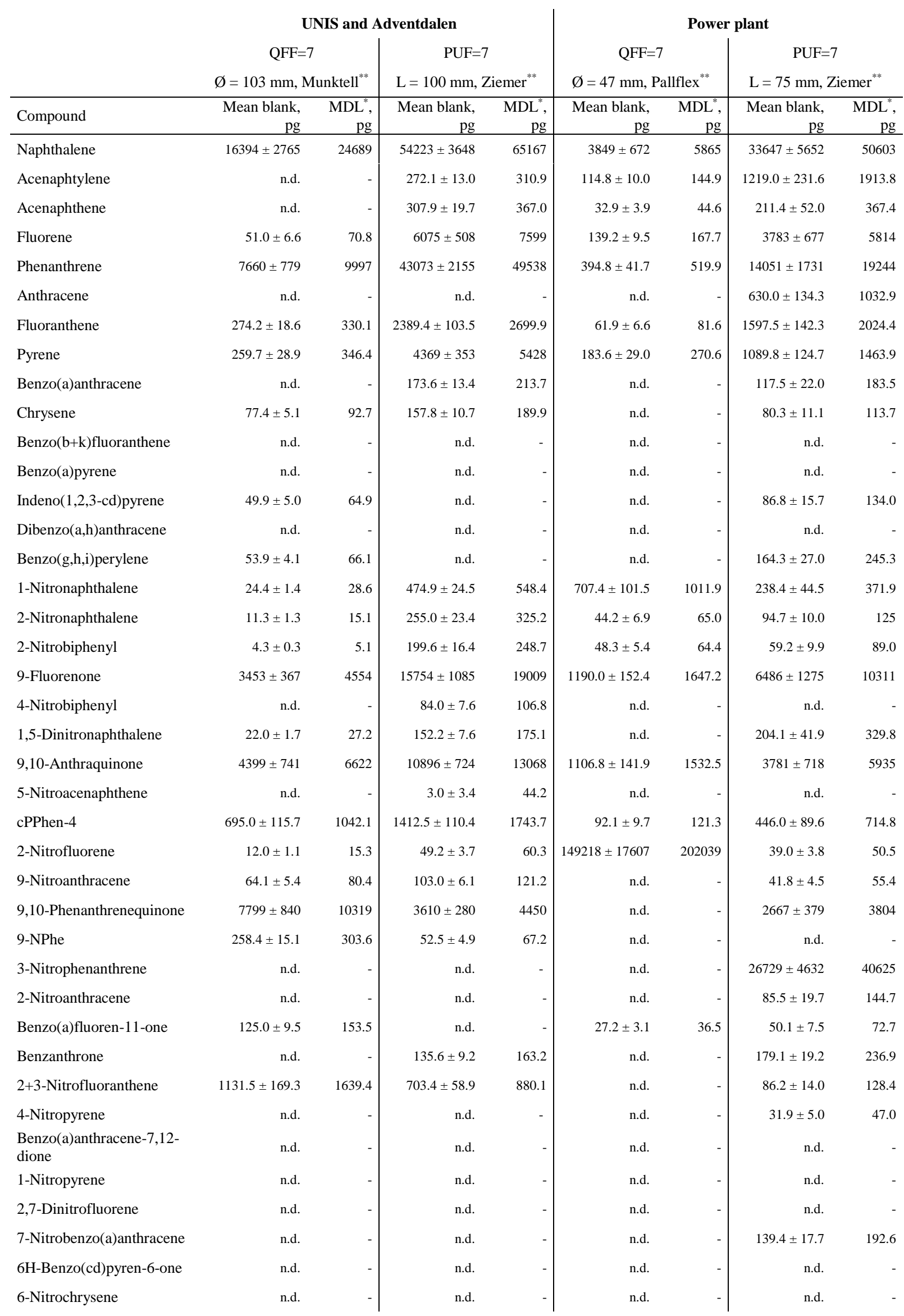




\begin{tabular}{|c|c|c|c|c|c|c|}
\hline 1,3-Dinitropyrene & n.d. & - & n.d. & n.d. & - & n.d. \\
\hline 1,6-Dinitropyrene & n.d. & - & n.d. & n.d. & - & n.d. \\
\hline 1,8-Dinitropyrene & n.d. & - & n.d. & n.d. & - & n.d. \\
\hline 6-Nitrobenzo(a)pyrene & n.d. & - & n.d. & n.d. & - & n.d. \\
\hline
\end{tabular}

${ }^{*} \mathrm{MDL}=$ blank $+3 * \mathrm{STD}$

** Sampling material with different size (PUF length or QFF diameter) and producers (Munktell, Ziemer, Pallflex) were used for air sampling

Table S7. Average recovery rates (Rec, \%) and relative standard deviations (RSTD, \%) for spiked samples

\begin{tabular}{|c|c|c|c|c|}
\hline \multirow[b]{2}{*}{ Compound } & \multicolumn{2}{|c|}{ Spiked QFF=4 } & \multicolumn{2}{|c|}{ Spiked PUF=4 } \\
\hline & Mean Rec, \% & RSTD, \% & Mean Rec, \% & RSTD, \% \\
\hline Nap & $128.6 \pm 10.0$ & 7.8 & $121.2 \pm 9.5$ & 7.8 \\
\hline Acy & $79.1 \pm 6.8$ & 8.6 & $93.3 \pm 1.6$ & 1.7 \\
\hline Ace & $46.2 \pm 4.2$ & 9.1 & $48.0 \pm 9.9$ & 20.5 \\
\hline Flu & $80.1 \pm 9.1$ & 11.3 & $109 \pm 13.4$ & 12.3 \\
\hline Phe & $78.2 \pm 9.4$ & 12.0 & $83.7 \pm 3.5$ & 4.1 \\
\hline Ant & $97.5 \pm 4.6$ & 4.7 & $119.8 \pm 4.2$ & 3.5 \\
\hline Flt & $37.5 \pm 5.4$ & 14.3 & $43.5 \pm 5.1$ & 11.6 \\
\hline Pyr & $80.9 \pm 4.4$ & 5.4 & $100.7 \pm 2.3$ & 2.3 \\
\hline BaAnt & $77.6 \pm 5.8$ & 7.5 & $90.3 \pm 4.3$ & 4.8 \\
\hline Chry & $78.9 \pm 9.1$ & 11.6 & $77.9 \pm 5.8$ & 7.4 \\
\hline BbkFlt & $72.4 \pm 10.1$ & 13.9 & $90.9 \pm 1.5$ & 1.7 \\
\hline BaPyr & $76.5 \pm 5.1$ & 6.6 & $86.0 \pm 13.6$ & 15.8 \\
\hline IPyr & $75.6 \pm 8.4$ & 11.1 & $78.2 \pm 5.7$ & 7.3 \\
\hline DBAnt & $86.9 \pm 11.1$ & 12.8 & $89.1 \pm 2.1$ & 2.3 \\
\hline BPer & $81.1 \pm 3.1$ & 3.8 & $94.6 \pm 10.0$ & 10.6 \\
\hline dNap & $64.5 \pm 3.4$ & 5.2 & $53.7 \pm 11.0$ & 20.4 \\
\hline dAcy & $90.1 \pm 9.5$ & 10.6 & $73.0 \pm 1.3$ & 1.8 \\
\hline dAce & $97.1 \pm 8.7$ & 9.0 & $89.4 \pm 11.1$ & 12.5 \\
\hline dFlu & $68.9 \pm 4.3$ & 6.2 & $76.2 \pm 9.3$ & 12.2 \\
\hline dPhe & $68.2 \pm 16.4$ & 24.0 & $82.4 \pm 17.2$ & 20.9 \\
\hline dAnt & $111.3 \pm 7.0$ & 6.3 & $97.3 \pm 5.3$ & 5.4 \\
\hline dFlt & $81.9 \pm 10.9$ & 13.3 & $100.4 \pm 22.2$ & 22.1 \\
\hline dPyr & $107.8 \pm 11.0$ & 10.2 & $76.8 \pm 5.0$ & 6.5 \\
\hline dBaAnt & $66.9 \pm 9.0$ & 13.4 & $82.6 \pm 27.9$ & 33.8 \\
\hline dChry & $69.5 \pm 9.7$ & 13.9 & $80.1 \pm 11.3$ & 14.1 \\
\hline dBbkFlt & $98.0 \pm 7.1$ & 7.3 & $100.6 \pm 15.7$ & 15.6 \\
\hline dBaPyr & $98.9 \pm 7.4$ & 7.4 & $98.7 \pm 5.9$ & 6.0 \\
\hline dIPyr & $102.2 \pm 15.9$ & 15.6 & $101.0 \pm 17.6$ & 17.5 \\
\hline dDBAnt & $93.2 \pm 8.4$ & 9.0 & $89.8 \pm 17.0$ & 19.0 \\
\hline dBPer & $96.9 \pm 17.2$ & 17.8 & $91.1 \pm 9.2$ & 10.1 \\
\hline 1-Nnap & $51.2 \pm 10.9$ & 21.2 & $72.3 \pm 7.1$ & 9.8 \\
\hline 2-Nnap & $61.4 \pm 7.5$ & 12.2 & $73.3 \pm 19.5$ & 26.6 \\
\hline 2-NBip & $74.4 \pm 16.8$ & 22.6 & $99.5 \pm 9.9$ & 9.9 \\
\hline 9-Flu & $40.1 \pm 2.0$ & 5.1 & $91.5 \pm 7.7$ & 8.4 \\
\hline 4-NBip & $65.9 \pm 4.4$ & 6.7 & $84.5 \pm 18.3$ & 21.7 \\
\hline
\end{tabular}




\begin{tabular}{|c|c|c|c|c|}
\hline 1,5-DNNap & $53.1 \pm 8.4$ & 15.9 & $69.0 \pm 7.1$ & 10.3 \\
\hline 9,10-AntQ & $42.0 \pm 6.9$ & 16.4 & $43.2 \pm 4.4$ & 10.3 \\
\hline 5-NAce & $74.3 \pm 4.9$ & 6.5 & $80.2 \pm 10.0$ & 12.5 \\
\hline cPPhe-4 & $44.1 \pm 6.5$ & 14.7 & $48.2 \pm 2.4$ & 4.9 \\
\hline 2-Nflu & $66.6 \pm 5.0$ & 7.6 & $66.3 \pm 14.9$ & 22.4 \\
\hline 9-Nant & $42.5 \pm 16.7$ & 39.3 & $78.0 \pm 6.7$ & 8.5 \\
\hline 9,10-PheQ & $56.8 \pm 10.2$ & 18.0 & $74.0 \pm 7.6$ & 10.3 \\
\hline 9-NPhe & $64.2 \pm 6.0$ & 9.3 & $104.7 \pm 15.6$ & 14.8 \\
\hline 3-Nphe & $65.7 \pm 8.4$ & 12.9 & $87.7 \pm 4.9$ & 5.5 \\
\hline 2-Nant & $46.2 \pm 11.6$ & 25.1 & $107.1 \pm 11.0$ & 10.3 \\
\hline BaFlu-11 & $47.4 \pm 3.0$ & 6.3 & $109.5 \pm 20.4$ & 18.6 \\
\hline BZT & $37.8 \pm 7.2$ & 19.0 & $43.8 \pm 3.1$ & 7.1 \\
\hline $2+3-\mathrm{NFlt}$ & $59.3 \pm 15.4$ & 25.9 & $79.6 \pm 13.5$ & 17.0 \\
\hline 4-Npyr & $54.6 \pm 5.7$ & 10.4 & $79.2 \pm 10.3$ & 13.0 \\
\hline BaAnt-7,12 & $46.8 \pm 12.1$ & 25.8 & $69.4 \pm 4.0$ & 5.8 \\
\hline 1-Npyr & $49.1 \pm 15.5$ & 31.5 & $56.2 \pm 7.9$ & 14.0 \\
\hline 7-NBaAnt & $69.8 \pm 35.2$ & 50.4 & $100.4 \pm 8.5$ & 8.5 \\
\hline BPyr-6 & $2.0 \pm 0.3$ & 13.7 & $4.1 \pm 0.2$ & 5.0 \\
\hline 6-Nchry & $58.8 \pm 5.3$ & 8.9 & $83.5 \pm 8.3$ & 9.9 \\
\hline 1,3-DNPyr & $17.8 \pm 3.0$ & 17.0 & $104.4 \pm 36.8$ & 35.2 \\
\hline 1,6-DNPyr & $22.6 \pm 1.0$ & 4.2 & $98.6 \pm 17.9$ & 18.2 \\
\hline 1.8-DNPyr & $9.5 \pm 0.3$ & 3.2 & $50.0 \pm 12.3$ & 24.5 \\
\hline 6-NBaPyr & $6.4 \pm 0.5$ & 7.1 & $32.6 \pm 7.0$ & 21.5 \\
\hline 1-NNap-d7 & $60.7 \pm 1.1$ & 1.7 & $73.3 \pm 9.2$ & 12.5 \\
\hline 2-NBP-d9 & $77.1 \pm 3.9$ & 5.1 & $104.1 \pm 12.6$ & 12.1 \\
\hline 9-Flu-d8 & $40.7 \pm 5.7$ & 13.9 & $73.8 \pm 8.8$ & 12.0 \\
\hline AntQ-d8 & $39.6 \pm 3.4$ & 8.5 & $50.3 \pm 8.6$ & 17.2 \\
\hline 2-NFlu-d9 & $68.6 \pm 9.7$ & 14.2 & $73.5 \pm 6.6$ & 8.9 \\
\hline 9-NAnt-d9 & $39.9 \pm 3.4$ & 8.6 & $69.0 \pm 8.3$ & 12.0 \\
\hline 3-NFlt-d9 & $55.3 \pm 7.6$ & 13.7 & $79.6 \pm 14.4$ & 18.1 \\
\hline 6-NChry-d11 & $59.4 \pm 1.2$ & 2.1 & $93.1 \pm 16.2$ & 17.4 \\
\hline
\end{tabular}


Table S8. Average recovery rates (Rec, \%) of internal standards (ISTDs) for ambient air samples

\begin{tabular}{lll} 
ISTD & Rec, $\%$ & Rec, $\%$ \\
& QFF $=15$ & PUF $=15$ \\
\hline dNap & $68.6 \pm 11.2$ & $68.4 \pm 6.2$ \\
dAcy & $70.7 \pm 7.8$ & $99.1 \pm 22.3$ \\
dAce & $100.2 \pm 27.5$ & $74.4 \pm 17.9$ \\
dFlu & $90.8 \pm 9.2$ & $76.9 \pm 25.8$ \\
dPhe & $66.3 \pm 5.8$ & $100.8 \pm 15.9$ \\
dAnt & $63.3 \pm 11.3$ & $91.7 \pm 33.0$ \\
dFlt & $68.6 \pm 5.2$ & $93.9 \pm 15.8$ \\
dPyr & $102.4 \pm 6.1$ & $84.3 \pm 11.6$ \\
dBaAnt & $94.8 \pm 12.6$ & $103.5 \pm 21.3$ \\
dChry & $63.9 \pm 7.6$ & $83.2 \pm 18.7$ \\
dBbkFlt & $99.8 \pm 15.4$ & $99.3 \pm 6.4$ \\
dBaPyr & $74.9 \pm 6.0$ & $97.5 \pm 17.0$ \\
dIPyr & $72.0 \pm 4.9$ & $109.1 \pm 20.0$ \\
dDBAnt & $80.1 \pm 22.6$ & $101.3 \pm 13.1$ \\
dBPer & $91.1 \pm 14.3$ & $79.3 \pm 12.9$ \\
1-NNap-d7 & $59.8 \pm 3.7$ & $80.3 \pm 23.7$ \\
2-NBP-d9 & $70.0 \pm 8.0$ & $89.0 \pm 4.3$ \\
9-Flu-d8 & $60.6 \pm 16.6$ & $67.7 \pm 4.3$ \\
9,10-AntQ-d8 & $56.2 \pm 12.4$ & $69.0 \pm 20.9$ \\
2-NFlu-d9 & $52.7 \pm 6.0$ & $84.5 \pm 18.1$ \\
9-NAnt-d9 & $74.5 \pm 14.8$ & $65.2 \pm 6.7$ \\
3-NFlt-d9 & $63.1 \pm 5.7$ & $49.3 \pm 7.4$ \\
6-NChry-d11 & $44.0 \pm 7.7$ & $49.1 \pm 9.0$ \\
\hline
\end{tabular}


Table S9. Concentrations of PAHs (G+P) and percentage in the particulate phase (\%PM) in Longyearbyen power plant $(\mathrm{n}=6)$, as well as MDLs for gaseous (PUF) and particulate $(\mathrm{QFF})$ phases, and instrumental LOD and LOQ; all values are in $\mathrm{pg}^{-3 *}$

\begin{tabular}{|c|c|c|c|c|c|c|c|c|}
\hline Variable & $\begin{array}{r}\text { Mean conc } \\
\pm \text { STD, pg m } \\
\end{array}$ & $\begin{array}{r}\text { Median } \\
\mathrm{pg} \mathrm{m}^{-3}\end{array}$ & $\begin{array}{r}\text { Min }- \text { Max } \\
\operatorname{pg~m}^{-3}\end{array}$ & $\begin{array}{l}\text { Mean } \\
\% \mathrm{PM}\end{array}$ & $\begin{array}{r}\mathrm{MDL}_{\mathrm{QFF}} \\
\mathrm{pg} \mathrm{m}^{-3}\end{array}$ & $\begin{array}{r}\text { MDLPUF }_{\text {PU m}}^{-3} \\
\mathrm{pg} \mathrm{m}^{-}\end{array}$ & $\begin{array}{r}\mathrm{LOD} \\
\mathrm{pg} \mathrm{m}^{-3}\end{array}$ & $\begin{array}{r}\text { LOQ } \\
\text { pg m }^{-3}\end{array}$ \\
\hline Naphthalene & $51817.0 \pm 10297.0$ & 55629.0 & $32737.0-59824.0$ & 7.4 & 1515.2 & 23992.0 & 0.3 & 0.9 \\
\hline Acenaphtylene & $2301.0 \pm 1059.0$ & 2203.0 & $1218.0-3797.0$ & 0.6 & 107.9 & 1024.0 & 0.2 & 0.6 \\
\hline Acenaphthene & $873.0 \pm 778.0$ & 487.0 & $300.0-2177.0$ & 8.4 & 29.5 & 145.2 & 0.2 & 0.6 \\
\hline Fluorene & $7607.0 \pm 3568.0$ & 6946.0 & $3680.0-12164.0$ & 4.6 & 62.3 & 3445.5 & 0.2 & 0.6 \\
\hline Phenanthrene & $27324.0 \pm 12700.0$ & 27530.0 & $12015.0-44871.0$ & 5.6 & 159.2 & 9242.6 & 0.2 & 0.7 \\
\hline Anthracene & $1055.0 \pm 818.0$ & 925.0 & $229.2-2136.0$ & 0.0 & n.d. & 458.4 & 0.3 & 0.8 \\
\hline Fluoranthene & $6991.0 \pm 3754.0$ & 6961.0 & $1428.0-12494.0$ & 3.9 & 47.7 & 1126.6 & 0.2 & 0.7 \\
\hline Pyrene & $4404.0 \pm 2178.0$ & 4700.0 & $1083.0-7348.0$ & 8.3 & 75.2 & 717.9 & 0.2 & 0.5 \\
\hline Benzo(a)anthracene & $130.7 \pm 77.3$ & 156.7 & $36.4^{2)}-202.8$ & 0.0 & n.d. & 72.9 & 0.6 & 1.7 \\
\hline Chrysene & $277.3 \pm 141.4$ & 333.3 & $60.7-421.3$ & 0.0 & n.d. & 54.7 & 0.5 & 1.4 \\
\hline Benzo(b+k)fluoranthene & n.d. & n.d. & n.d. & - & n.d. & n.d. & 0.5 & 1.4 \\
\hline Benzo(a)pyrene & n.d. & n.d. & n.d. & - & n.d. & n.d. & 0.5 & 1.5 \\
\hline Indeno(1,2,3-cd)pyrene & n.d. & n.d. & n.d. & - & n.d. & 132.2 & 0.6 & 1.8 \\
\hline Dibenzo(a,h)anthracene & n.d. & n.d. & n.d. & - & n.d. & n.d. & 0.6 & 1.8 \\
\hline Benzo(g,h,i)perylene & n.d. & n.d. & n.d. & - & n.d. & 225.4 & 0.6 & 1.7 \\
\hline 1-Nitronaphthalene & $2188.0 \pm 1341.0$ & 1729.0 & $988.0-4685.0$ & 61.7 & 241.0 & 158.9 & 0.0 & 0.0 \\
\hline 2-Nitronaphthalene & $257.3 \pm 108.8$ & 273.6 & $105.9-395.1$ & 31.7 & 20.3 & 74.9 & 0.0 & 0.0 \\
\hline 2-Nitrobiphenyl & $157.4 \pm 75.7$ & 154.2 & $69.2-290.3$ & 39.9 & 18.8 & 38.8 & 0.0 & 0.1 \\
\hline 9-Fluorenone & $12352.0 \pm 5258.0$ & 11937.0 & $5566.0-19541.0$ & 19.2 & 479.0 & 4368.6 & 0.1 & 0.2 \\
\hline 4-Nitrobiphenyl & n.d. & n.d. & n.d. & - & n.d. & n.d. & 0.0 & 0.1 \\
\hline 1,5-Dinitronaphthalene & n.d. & n.d. & n.d. & - & n.d. & 139.7 & 0.0 & 0.1 \\
\hline 9,10-Anthraquinone & $15758.0 \pm 15578.0$ & 10806.0 & $4595.0-46956.0$ & 21.3 & 446.8 & 2558.7 & 0.1 & 0.4 \\
\hline 5-Nitroacenaphthene & n.d. & n.d. & n.d. & - & n.d. & n.d. & 0.0 & 0.1 \\
\hline cPPhen-4 & $1304.0 \pm 743.0$ & 1320.0 & $506.0-2554.0$ & 15.8 & 39.3 & 326.7 & 0.1 & 0.2 \\
\hline 2-Nitrofluorene & $42.5 \pm 54.3$ & 25.0 & $22.0^{2)}-134.5$ & 0.0 & 777.5 & 44.0 & 0.0 & 0.1 \\
\hline 9-Nitroanthracene & $79.6 \pm 77.2$ & 61.4 & $20.5^{2)}-226.7$ & 0.0 & n.d. & 41.1 & 0.1 & 0.2 \\
\hline 9,10-Phenanthrenequinone & $2127.0 \pm 1934.0$ & 2061.0 & $960.1^{2)}-4400.0$ & 0.0 & n.d. & 1920.2 & 9.4 & 28.3 \\
\hline 9-Nitrophenanthrene & n.d. & n.d. & n.d. & - & n.d. & n.d. & 0.1 & 0.2 \\
\hline 3-Nitrophenanthrene & $761.0 \pm 757.0$ & 656.0 & $0.3^{1)}-1930.0$ & 96.1 & n.d. & 76.0 & 0.1 & 0.3 \\
\hline 2-Nitroanthracene & $305.7 \pm 235.5$ & 261.4 & $66.1^{2)}-620.9$ & 0.0 & n.d. & 132.2 & 0.1 & 0.3 \\
\hline Benzo(a)fluoren-11-one & $157.5 \pm 55.2$ & 165.0 & $75.5-227.0$ & 27.6 & 27.0 & 32.9 & 0.1 & 0.4 \\
\hline Benzanthrone & $866.0 \pm 418.0$ & 920.0 & $135.0-1314.0$ & 0.0 & n.d. & 117.1 & 0.4 & 1.3 \\
\hline $2+3$-Nitrofluoranthene & $519.3 \pm 437.1$ & 442.1 & $61.0-1140.3$ & 0.0 & n.d. & 53.6 & 0.2 & 0.6 \\
\hline 4-Nitropyrene & $114.8 \pm 70.0$ & 151.5 & $25.7^{2)}-174.5$ & 0.0 & n.d. & 51.4 & 0.2 & 0.6 \\
\hline Benzo(a)anthracene-7,12-dione & n.d. & n.d. & n.d. & - & n.d. & n.d. & 0.3 & 0.8 \\
\hline 1-Nitropyrene & n.d. & n.d. & n.d. & - & n.d. & n.d. & 0.2 & 0.6 \\
\hline 2,7-Dinitrofluorene & $58.8 \pm 53.1$ & 70.5 & $0.6^{1)}-141.0$ & 0.0 & n.d. & n.d. & 0.2 & 0.6 \\
\hline 7-Nitrobenzo(a)anthracene & $578.0 \pm 376.0$ & 709.0 & $114.2^{2)}-932.0$ & 0.0 & n.d. & 228.5 & 0.2 & 0.6 \\
\hline 6H-Benzo(cd)pyren-6-one & n.d. & n.d. & n.d. & - & n.d. & n.d. & 0.9 & 2.6 \\
\hline 6-Nitrochrysene & n.d. & n.d. & n.d. & - & n.d. & n.d. & 0.2 & 0.6 \\
\hline 1,3-Dinitropyrene & n.d. & n.d. & n.d. & - & n.d. & n.d. & 0.2 & 0.6 \\
\hline 1,6-Dinitropyrene & n.d. & n.d. & n.d. & - & n.d. & n.d. & 0.3 & 1.0 \\
\hline 1,8-Dinitropyrene & n.d. & n.d. & n.d. & - & n.d. & n.d. & 0.3 & 0.8 \\
\hline 6-Nitrobenzo(a)pyrene & n.d. & n.d. & n.d. & - & n.d. & n.d. & 0.2 & 0.6 \\
\hline$\sum$ PAHs & $102846 \pm 31931$ & & $61925-139082$ & & & & & \\
\hline$\sum$ oxy-PAHs & $32565 \pm 20963$ & & $15767-73084$ & & & & & \\
\hline$\sum$ nitro-PAHs & $4451 \pm 2075$ & & $2046-7752$ & & & & & \\
\hline
\end{tabular}


Table S10. Concentrations of PAHs $(\mathrm{G}+\mathrm{P})$ and percentage in the particulate phase (\%PM) at $U N I S(n=6)$, as well as MDLs for gaseous (PUF) and particulate (QFF) phases, and instrumental LOD and LOQ; all values are in $\mathrm{pg} \mathrm{m}^{-3 *}$

\begin{tabular}{|c|c|c|c|c|c|c|c|c|}
\hline Variable & $\begin{array}{r}\text { Mean conc } \\
\pm \mathrm{STD}, \mathrm{pg} \mathrm{m}^{-3} \\
\end{array}$ & $\begin{array}{c}\text { Median } \\
\mathrm{pg} \mathrm{m}^{-3}\end{array}$ & $\begin{array}{r}\text { Min }- \text { Max } \\
\operatorname{pg~m}^{-3}\end{array}$ & $\begin{array}{r}\text { Mean \%PM } \\
\pm \text { STD }\end{array}$ & $\begin{array}{l}\text { MDL qff } \\
\operatorname{pg~m}^{-3}\end{array}$ & $\begin{array}{r}\text { MDL puf } \\
\operatorname{pg~m}^{-3}\end{array}$ & $\begin{array}{r}\text { LOD } \\
\mathrm{pg} \mathrm{m}^{-3}\end{array}$ & $\begin{array}{r}\mathrm{LOQ} \\
\mathrm{pg} \mathrm{m}^{-3} \\
\end{array}$ \\
\hline Naphthalene & $<\mathrm{MDL}$ & $<\mathrm{MDL}$ & $<\mathrm{MDL}$ & - & 45.6 & 241.8 & 0.002 & 0.005 \\
\hline Acenaphtylene & $16.9 \pm 8.2$ & 16.6 & $7.1-29.2$ & $0 \pm 0.0$ & n.d. & 0.8 & 0.001 & 0.003 \\
\hline Acenaphthene & $48.5 \pm 20.5$ & 45.7 & $24.3-73.0$ & $0 \pm 0.0$ & n.d. & 0.9 & 0.001 & 0.003 \\
\hline Fluorene & $170.5 \pm 39.6$ & 155.2 & $136.5-236.0$ & $1.1 \pm 1.0$ & 0.2 & 26.6 & 0.001 & 0.003 \\
\hline Phenanthrene & $409.2 \pm 34.3$ & 401.8 & $368.5-470.0$ & $6.5 \pm 8.6$ & 32.4 & 179.6 & 0.001 & 0.004 \\
\hline Anthracene & $18.0 \pm 4.5$ & 17.2 & $12.3-25.5$ & $0 \pm 0.0$ & n.d. & n.d. & 0.001 & 0.004 \\
\hline Fluoranthene & $28.5 \pm 4.3$ & 27.5 & $24.1-36.1$ & $40.5 \pm 19.4$ & 1.2 & 7.4 & 0.001 & 0.004 \\
\hline Pyrene & $39.5 \pm 6.3$ & 37.9 & $30.7-47.8$ & $26.8 \pm 18.6$ & 1.1 & 12.7 & 0.001 & 0.003 \\
\hline Benzo(a)anthracene & $2.2 \pm 2.6$ & 1.2 & $0.009^{1)}-5.8$ & $68.2 \pm 2.4$ & n.d. & 0.5 & 0.003 & 0.009 \\
\hline Chrysene & $7.3 \pm 4.3$ & 6.6 & $2.6-13.5$ & $81.7 \pm 10.3$ & 0.2 & 0.4 & 0.003 & 0.008 \\
\hline Benzo(b+k)fluoranthene & $2.2 \pm 2.1$ & 1.8 & $0.008^{1)}-5.9$ & $100 \pm 0.0$ & n.d. & n.d. & 0.003 & 0.008 \\
\hline Benzo(a)pyrene & $0.9 \pm 1.1$ & 0.6 & $0.008^{1)}-2.5$ & $100 \pm 0.0$ & n.d. & n.d. & 0.003 & 0.008 \\
\hline Indeno(1,2,3-cd)pyrene & $1.6 \pm 1.5$ & 1.8 & $0.07^{2)}-3.8$ & $100 \pm 0.0$ & 0.15 & n.d. & 0.003 & 0.010 \\
\hline Dibenzo(a,h)anthracene & n.d. & n.d. & n.d. & - & n.d. & n.d. & 0.003 & 0.009 \\
\hline Benzo(g,h,i)perylene & $3.9 \pm 2.5$ & 3.7 & $1.4-8.1$ & $100 \pm 0.0$ & 0.2 & n.d. & 0.003 & 0.009 \\
\hline 1-Nitronaphthalene & $17.0 \pm 3.0$ & 16.4 & $13.4-21.5$ & $0.1 \pm 0.2$ & 0.1 & 1.3 & 0.000 & 0.001 \\
\hline 2-Nitronaphthalene & $5.1 \pm 2.0$ & 5.1 & $2.4-7.3$ & $3.1 \pm 5.6$ & 0.04 & 1.0 & 0.000 & 0.001 \\
\hline 2-Nitrobiphenyl & $1.0 \pm 0.1$ & 1.0 & $0.8-1.2$ & $10.1 \pm 8.3$ & 0.01 & 0.6 & 0.000 & 0.001 \\
\hline 9-Fluorenone & $270.3 \pm 146.9$ & 211.2 & $128.2-543.8$ & $41.7 \pm 22.3$ & 14.1 & 62.1 & 0.001 & 0.004 \\
\hline 4-Nitrobiphenyl & $2.2 \pm 0.5$ & 2.4 & $1.5-2.7$ & $0 \pm 0.0$ & n.d. & 0.2 & 0.001 & 0.002 \\
\hline 1,5-Dinitronaphthalene & $0.8 \pm 0.9$ & 0.5 & $0.05-2.2$ & $80.0 \pm 44.7$ & 0.1 & 0.4 & 0.001 & 0.002 \\
\hline 9,10-Anthraquinone & $163.5 \pm 57.4$ & 159.6 & $105.2-269.1$ & $37.5 \pm 22.4$ & 19.3 & 22.8 & 0.002 & 0.007 \\
\hline 5-Nitroacenaphthene & $0.2 \pm 0.1$ & 0.2 & $0.05^{2)}-0.38$ & - & n.d. & 0.1 & 0.001 & 0.002 \\
\hline cPPhen-4 & $27.2 \pm 6.9$ & 25.1 & $20.2-35.8$ & $65.5 \pm 10.6$ & 2.8 & 5.1 & 0.001 & 0.004 \\
\hline 2-Nitrofluorene & $0.2 \pm 0.3$ & 0.1 & $0.07^{2)}-0.8$ & $15.1 \pm 7.5$ & 0.04 & 0.15 & 0.001 & 0.002 \\
\hline 9-Nitroanthracene & $0.6 \pm 0.3$ & 0.7 & $0.2^{2)}-0.9$ & - & 0.2 & 0.4 & 0.001 & 0.003 \\
\hline 9,10-Phenanthrenequinone & $<\mathrm{MDL}$ & $<\mathrm{MDL}$ & $<\mathrm{MDL}$ & - & 22.5 & 14.4 & 0.155 & 0.464 \\
\hline 9-Nitrophenanthrene & $0.2 \pm 0.2$ & 0.3 & $0.1^{2)}-0.4$ & - & 1.1 & 0.2 & 0.001 & 0.003 \\
\hline 3-Nitrophenanthrene & n.d. & n.d. & n.d. & - & n.d. & n.d. & 0.002 & 0.005 \\
\hline 2-Nitroanthracene & n.d. & n.d. & n.d. & - & n.d. & n.d. & 0.002 & 0.005 \\
\hline Benzo(a)fluoren-11-one & $6.1 \pm 3.6$ & 5.0 & $1.8-11.1$ & $100 \pm 0.0$ & 0.6 & n.d. & 0.002 & 0.006 \\
\hline Benzanthrone & $1.8 \pm 1.8$ & 1.7 & $0.02^{1)}-4.3$ & $96.7 \pm 4.8$ & n.d. & 0.5 & 0.007 & 0.022 \\
\hline 2+3-Nitrofluoranthene & $9.5 \pm 1.6$ & 9.7 & $7.3-11.4$ & $94.5 \pm 13.5$ & 4.5 & 2.1 & 0.003 & 0.010 \\
\hline 4-Nitropyrene & n.d. & n.d. & n.d. & - & n.d. & n.d. & 0.003 & 0.009 \\
\hline Benzo(a)anthracene-7,12-dione & $2.2 \pm 1.8$ & 2.2 & $0.01^{1)}-4.9$ & $100 \pm 0.0$ & n.d. & n.d. & 0.004 & 0.013 \\
\hline 1-Nitropyrene & n.d. & n.d. & n.d. & - & n.d. & n.d. & 0.003 & 0.010 \\
\hline 2,7-Dinitrofluorene & n.d. & n.d. & n.d. & - & n.d. & n.d. & 1.003 & 3.010 \\
\hline 7-Nitrobenzo(a)anthracene & n.d. & n.d. & n.d. & - & n.d. & n.d. & 0.003 & 0.010 \\
\hline 6H-Benzo(cd)pyren-6-one & n.d. & n.d. & n.d. & - & n.d. & n.d. & 0.014 & 0.043 \\
\hline 6-Nitrochrysene & n.d. & n.d. & n.d. & - & n.d. & n.d. & 0.004 & 0.011 \\
\hline 1,3-Dinitropyrene & n.d. & n.d. & n.d. & - & n.d. & n.d. & 0.003 & 0.010 \\
\hline 1,6-Dinitropyrene & n.d. & n.d. & n.d. & - & n.d. & n.d. & 0.006 & 0.017 \\
\hline 1,8-Dinitropyrene & n.d. & n.d. & n.d. & - & n.d. & n.d. & 0.004 & 0.013 \\
\hline 6-Nitrobenzo(a)pyrene & n.d. & n.d. & n.d. & - & n.d. & n.d. & 0.003 & 0.009 \\
\hline$\sum$ PAHs & $749.2 \pm 72.6$ & & $687.4-866.9$ & & & & & \\
\hline $\bar{\sum}$ oxy-PAHs & $471.0 \pm 150.8$ & & $325.9-741.4$ & & & & & \\
\hline$\sum$ nitro-PAHs & $36.76 \pm 6.19$ & & $30.30-46.10$ & & & & & \\
\hline
\end{tabular}

* LOD, LOQ and MDL in pg (Tables S4-S6) were converted to $\mathrm{pg} \mathrm{m}^{-3}$ using $370 \mathrm{~m}^{3}$ as average sample volume

$<$ MDL below method detection limit

n.d. not detected

1) equal LOQ

2) $1 / 2$ of MDL 
Table S11. Concentrations of PAHs (G+P) and percentage in the particulate phase (\% PM) at Adventdalen $(n=6)$, as well as MDLs for gaseous (PUF) and particulate (QFF) phases, and instrumental LOD and LOQ; all values are in $\mathbf{p g ~} \mathrm{m}^{-3} *$

\begin{tabular}{|c|c|c|c|c|c|c|c|c|}
\hline Variable & $\begin{array}{l}\text { Mean conc } \\
\pm \text { STD, } \text { pg m }^{-3}\end{array}$ & $\begin{array}{r}\text { Median } \\
\text { pg m }^{-3}\end{array}$ & $\begin{array}{r}\text { Min }- \text { Max } \\
\operatorname{pg~m}^{-3} \\
\end{array}$ & $\begin{array}{r}\text { Mean \%PM } \\
\pm \text { STD } \\
\end{array}$ & $\begin{array}{r}\text { MDL qff } \\
\operatorname{pg~m}^{-3}\end{array}$ & $\begin{array}{r}\text { MDL puf } \\
\operatorname{pg~m}^{-3}\end{array}$ & $\begin{array}{r}\text { LOD } \\
\mathrm{pg} \mathrm{m}^{-3}\end{array}$ & $\begin{array}{r}\text { LOQ } \\
\mathrm{pg} \mathrm{m}^{-3}\end{array}$ \\
\hline Naphthalene & $<\mathrm{MDL}$ & $<\mathrm{MDL}$ & $<\mathrm{MDL}$ & - & 45.6 & 241.8 & 0.002 & 0.005 \\
\hline Acenaphtylene & $2.4 \pm 1.5$ & 2.1 & $1.1-5.1$ & $0 \pm 0$ & - & 0.8 & 0.001 & 0.004 \\
\hline Acenaphthene & $3.8 \pm 2.5$ & 3.8 & $1.3-6.6$ & $0 \pm 0$ & - & 0.9 & 0.001 & 0.003 \\
\hline Fluorene & $60.0 \pm 23.2$ & 50.1 & $38.5-95.8$ & $1.8 \pm 3.0$ & 0.2 & 26.6 & 0.001 & 0.003 \\
\hline Phenanthrene & $236.3 \pm 31.8$ & 236.2 & $191.7-270.8$ & $3.7 \pm 9.0$ & 32.4 & 179.6 & 0.001 & 0.004 \\
\hline Anthracene & $14.3 \pm 3.4$ & 13.9 & $10.5-19.3$ & $3.5 \pm 8.5$ & - & - & 0.001 & 0.004 \\
\hline Fluoranthene & $19.1 \pm 10.0$ & 15.8 & $10.8-38.5$ & $23.2 \pm 9.2$ & 1.2 & 7.4 & 0.001 & 0.004 \\
\hline Pyrene & $27.2 \pm 6.4$ & 26.4 & $20.9-35.9$ & $15.8 \pm 11.0$ & 1.1 & 12.7 & 0.001 & 0.003 \\
\hline Benzo(a)anthracene & n.d. & n.d. & n.d. & - & - & 0.5 & 0.003 & 0.009 \\
\hline Chrysene & $3.1 \pm 2.5$ & 2.7 & $0.1^{1)}-7.1$ & $64.1 \pm 18.7$ & 0.2 & 0.4 & 0.003 & 0.008 \\
\hline Benzo $(b+k)$ fluoranthene & $0.7 \pm 1.2$ & 0.0 & $0.008^{1)}-2.785$ & $100 \pm 0$ & - & - & 0.003 & 0.008 \\
\hline Benzo(a)pyrene & $0.3 \pm 0.5$ & 0.0 & $0.008^{1)}-1.163$ & $100 \pm 0$ & - & - & 0.003 & 0.008 \\
\hline Indeno(1,2,3-cd)pyrene & $0.7 \pm 1.2$ & 0.0 & $0.07^{2)}-2.67$ & $100 \pm 0$ & 0.15 & - & 0.003 & 0.010 \\
\hline Dibenzo(a,h)anthracene & n.d. & n.d. & n.d. & - & - & - & 0.003 & 0.010 \\
\hline Benzo(g,h,i)perylene & $1.2 \pm 1.5$ & 0.8 & $0.08^{2)}-3.83$ & $100 \pm 0$ & 0.2 & - & 0.003 & 0.009 \\
\hline 1-Nitronaphthalene & $5.0 \pm 3.2$ & 4.2 & $1.9-9.8$ & $1.5 \pm 3.6$ & 0.1 & 1.3 & 0.000 & 0.001 \\
\hline 2-Nitronaphthalene & $1.9 \pm 0.6$ & 1.7 & $1.3-2.8$ & $5.4 \pm 8.0$ & 0.1 & 1.0 & 0.000 & 0.001 \\
\hline 2-Nitrobiphenyl & $1.0 \pm 0.2$ & 0.9 & $0.8-1.3$ & $5.9 \pm 10.0$ & 0.1 & 0.6 & 0.000 & 0.001 \\
\hline 9-Fluorenone & $139.4 \pm 24.9$ & 137.3 & $110.2-177.2$ & $25.5 \pm 13.4$ & 14.1 & 62.1 & 0.001 & 0.004 \\
\hline 4-Nitrobiphenyl & $2.5 \pm 1.2$ & 2.6 & $0.3-4.1$ & - & - & 0.2 & 0.001 & 0.002 \\
\hline 1,5-Dinitronaphthalene & $0.9 \pm 1.4$ & 0.6 & $0.05^{2)}-3.72$ & $53.9 \pm 53.6$ & 0.1 & 0.4 & 0.001 & 0.002 \\
\hline 9,10-Anthraquinone & $71.7 \pm 39.2$ & 80.3 & $11.4^{2)}-118.4$ & $43.9 \pm 1.4$ & 19.3 & 22.8 & 0.002 & 0.007 \\
\hline 5-Nitroacenaphthene & $0.3 \pm 0.7$ & 0.0 & $0.05^{2)}-1.62$ & - & - & 0.1 & 0.001 & 0.002 \\
\hline cPPhen-4 & $18.8 \pm 10.2$ & 15.1 & $12.0-39.1$ & $38.1 \pm 20.2$ & 2.8 & 5.1 & 0.001 & 0.004 \\
\hline 2-Nitrofluorene & $0.6 \pm 0.4$ & 0.7 & $0.07^{2)}-1.05$ & $4.2 \pm 9.5$ & 0.15 & 0.1 & 0.001 & 0.002 \\
\hline 9-Nitroanthracene & $2.3 \pm 1.9$ & 1.8 & $1.0-4.7$ & $57.8 \pm 29.7$ & 0.2 & 0.4 & 0.001 & 0.003 \\
\hline 9,10-Phenanthrenequinone & n.d. & n.d. & n.d. & - & 22.5 & 14.4 & 0.155 & 0.464 \\
\hline 9-Nitrophenanthrene & $0.4 \pm 0.4$ & 0.4 & $0.09^{2)}-1.17$ & $25 \pm 50$ & 1.1 & 0.2 & 0.001 & 0.003 \\
\hline 3-Nitrophenanthrene & n.d. & n.d. & n.d. & - & - & - & 0.002 & 0.005 \\
\hline 2-Nitroanthracene & n.d. & n.d. & n.d. & - & - & - & 0.002 & 0.005 \\
\hline Benzo(a)fluoren-11-one & $2.2 \pm 1.7$ & 1.6 & $0.7-4.4$ & $100 \pm 0$ & 0.6 & - & 0.002 & 0.006 \\
\hline Benzanthrone & $0.1 \pm 0.2$ & 0.0 & $0.02^{1)}-0.58$ & $100 \pm 0$ & - & 0.5 & 0.007 & 0.022 \\
\hline 2+3-Nitrofluoranthene & $12.3 \pm 7.7$ & 9.8 & $4.7-26.7$ & $79.8 \pm 16.5$ & 4.5 & 2.1 & 0.003 & 0.010 \\
\hline 4-Nitropyrene & n.d. & n.d. & n.d. & - & - & - & 0.003 & 0.009 \\
\hline Benzo(a)anthracene-7,12-dione & $0.9 \pm 0.7$ & 0.7 & $0.01^{1)}-2.21$ & $100 \pm 0$ & - & - & 0.004 & 0.013 \\
\hline 1-Nitropyrene & n.d. & n.d. & n.d. & - & - & - & 0.003 & 0.010 \\
\hline 2,7-Dinitrofluorene & n.d. & n.d. & n.d. & - & - & - & 1.003 & 3.010 \\
\hline 7-Nitrobenzo(a)anthracene & n.d. & n.d. & n.d. & - & - & - & 0.003 & 0.010 \\
\hline 6H-Benzo(cd)pyren-6-one & n.d. & n.d. & n.d. & - & - & - & 0.015 & 0.043 \\
\hline 6-Nitrochrysene & n.d. & n.d. & n.d. & - & - & - & 0.004 & 0.011 \\
\hline 1,3-Dinitropyrene & n.d. & n.d. & n.d. & - & - & - & 0.003 & 0.010 \\
\hline 1,6-Dinitropyrene & n.d. & n.d. & n.d. & - & - & - & 0.006 & 0.017 \\
\hline 1,8-Dinitropyrene & n.d. & n.d. & n.d. & - & - & - & 0.004 & 0.013 \\
\hline 6-Nitrobenzo(a)pyrene & n.d. & n.d. & n.d. & - & - & - & 0.003 & 0.009 \\
\hline$\sum$ PAHs & $369.1 \pm 66.7$ & & $279.0-454.5$ & & & & & \\
\hline$\sum o x y-P A H s$ & $233.1 \pm 68.3$ & & $124.7-337.1$ & & & & & \\
\hline$\sum$ nitro-PAHs & $27.16 \pm 11.14$ & & $13.50-44.43$ & & & & & \\
\hline
\end{tabular}

* LOD, LOQ and MDL in pg (Tables S4-S6) were converted to $\mathrm{pg} \mathrm{m}^{-3}$ using $370 \mathrm{~m}^{3}$ as average sample volume

$<$ MDL below method detection limit

n.d. not detected

1) equal LOQ

2) $1 / 2$ of MDL 
Table S12. Comparison of Longyearbyen power plant 16 PAH emissions with other coal-burning plants operated worldwide

\begin{tabular}{|c|c|c|c|c|c|c|c|c|c|c|c|}
\hline Power plant & Location & Coal type & $\begin{array}{l}\text { Boiler capacity, } \\
\text { conditions }\end{array}$ & Flue gas cleaning & $\begin{array}{r}\text { Dust in } \\
\text { flue gas, } \\
\mathrm{mg} \mathrm{m}^{-3}\end{array}$ & $\begin{array}{r}\mathrm{EF}, \\
\mu \mathrm{g} \mathrm{kg}^{-1}\end{array}$ & $\underset{\mu \mathrm{gAHs} \mathrm{m}^{-3}}{\sum \text { PAH }}$ & $\% \mathrm{PM}$ & $\begin{array}{l}\text { Predominant } \\
\text { PAHs }\end{array}$ & PAH profile & References \\
\hline Energyverket & $\begin{array}{l}\text { Longyearbyen, } \\
\text { Svalbard }\end{array}$ & $\begin{array}{l}\text { Bituminous, } \\
\text { Ro }=0.78 \% \text {, } \\
\text { High volatile }\end{array}$ & $\begin{array}{l}68 \text { ton-coal/day, } \\
\text { two boilers } 32 \\
\text { MW each }\end{array}$ & SNCR+ESP+WFGD & 1.5 & 1.5 & 0.106 & 0.06 & $\begin{array}{l}\text { Nap+ Phe+ Flu, } \\
\text { Flt + Pyr }\end{array}$ & $\begin{array}{l}53 \% 2 \text { rings, } \\
36 \% 3 \text { rings, } \\
11 \% 4 \text { rings } \\
5-6 \text { rings n.d. }\end{array}$ & This work \\
\hline n.a. & Central Taiwan & $\begin{array}{l}\text { Indonesian } \\
\text { and } \\
\text { Australian }\end{array}$ & 6480 ton-coal/day & $\mathrm{SCR}+\mathrm{ESP}+\mathrm{FGD}$ & 6.0 & 1.5 & 0.268 & n.a. & $\begin{array}{l}\text { Nap + Phe, Flt, } \\
\text { Pyr + Flu }\end{array}$ & $\begin{array}{l}45 \% 2 \text { rings, } \\
20 \% 3 \text { rings, } \\
30 \% 4 \text { rings, } \\
5 \% \text { rest }\end{array}$ & (Hsu et al., 2016) \\
\hline Fusina plant & $\begin{array}{l}\text { Porto Marghera, } \\
\text { Italy }\end{array}$ & n.a. & n.a. & $\mathrm{SCR}+\mathrm{ESP}+\mathrm{FGD}$ & 5.2 & n.a. & 0.697 & n.a. & n.a. & n.a. & (Rigamonti et al., 2012) \\
\hline n.a. & Netherlands & $\begin{array}{l}\text { From } \\
\text { different } \\
\text { countries }\end{array}$ & n.a. & $\mathrm{ESP}+\mathrm{FGD}$ & n.a. & n.a. & $1-3$ & n.a. & n.a. & n.a. & $\begin{array}{l}\text { (Meij and Te Winkel, } \\
\text { 2007) }\end{array}$ \\
\hline Point $\mathrm{F}$ & China & Blend & $1000 \mathrm{MW}$ & $\begin{array}{l}\text { SCR + LLT-ESP + } \\
\text { WFGD }\end{array}$ & 4.0 & n.a. & 5.255 & 0.09 & $\begin{array}{l}\text { Nap+ Phe } \\
+6 \text { rings }\end{array}$ & $80 \% 2$ rings & (Li et al., 2016) \\
\hline Point $\mathrm{G}$ & China & Blend & $1000 \mathrm{MW}$ & $\begin{array}{l}\text { SCR + LLT-ESP + } \\
\text { WFGD + WESP }\end{array}$ & $<1.9$ & n.a. & 0.870 & 0.51 & $\begin{array}{l}5 \text { and } 6 \text { rings, } \\
\text { no Nap }\end{array}$ & $\begin{array}{l}73 \% 5 \text { and } 6 \\
\text { rings }\end{array}$ & (Li et al., 2016) \\
\hline HPA-3 & Huainan, China & Bituminous & $600 \mathrm{MW}$ & ESP + WFGD & 10.4 & n.a. & 11.67 & 0.39 & $\begin{array}{l}\text { Nap + Acy, Ant, } \\
\text { Phe }\end{array}$ & n.a. & (Wang et al., 2015) \\
\hline HPB-2 & Huainan, China & Bituminous & $600 \mathrm{MW}$ & $\mathrm{ESP}+\mathrm{WFGD}$ & 10.6 & n.a. & 11.87 & 0.30 & Nap + Acy, Flu & n.a. & (Wang et al., 2015) \\
\hline HPC-1 & Huainan, China & Bituminous & $600 \mathrm{MW}$ & $\mathrm{ESP}+\mathrm{WFGD}$ & 11.2 & n.a. & 8.84 & 0.32 & Nap + Acy, Ant & n.a. & (Wang et al., 2015) \\
\hline
\end{tabular}

260 n.a. not available

SCR is selective catalytic reduction system; SNCR is selective non-catalytic reduction system

ESP is electrostatic precipitator; LLT-ESP is low-low temperature ESP

FGD is flue-gas desulfurization scrubber; WFGD is wet FGD 
Table S13. UNIS and Adventdalen air concentrations (G+P) of 16 PAHs compared to national and regional background concentrations detected in autumn 2018

\begin{tabular}{|c|c|c|c|c|c|c|c|c|c|c|c|c|}
\hline \multirow{2}{*}{ Components } & \multicolumn{3}{|c|}{ UNIS $\left(\mathrm{ng} \mathrm{m}^{-3}\right)^{2)}$} & \multicolumn{3}{|c|}{ Adventdalen $\left(\mathrm{ng} \mathrm{m}^{-3}\right)^{2)}$} & \multicolumn{3}{|c|}{ Birkenes $\left(\mathrm{ng} \mathrm{m}^{-3}\right)^{1,3)}$} & \multicolumn{3}{|c|}{ Zeppelin $\left(\mathrm{ng} \mathrm{m}^{-3}\right)^{1,4)}$} \\
\hline & Average & Min & Max & Average & Min & Max & Average & Min & Max & Average & Min & Max \\
\hline Naphthalene & n.q. & n.q. & n.q. & n.q. & n.q. & n.q. & 0.034 & 0.028 & 0.044 & $0.094^{5)}$ & 0.081 & 0.107 \\
\hline Acenaphthylene & 0.017 & 0.007 & 0.029 & 0.002 & 0.001 & 0.002 & 0.019 & 0.002 & 0.052 & n.d. & n.d. & n.d. \\
\hline Acenaphthene & 0.049 & 0.024 & 0.073 & 0.004 & 0.001 & 0.007 & 0.141 & 0.027 & 0.301 & 0.002 & 0.002 & 0.002 \\
\hline Fluorene & 0.171 & 0.137 & 0.236 & 0.060 & 0.039 & 0.096 & 0.264 & 0.172 & 0.334 & 0.009 & 0.001 & 0.014 \\
\hline Phenanthrene & 0.409 & 0.369 & 0.470 & 0.236 & 0.192 & 0.271 & 0.675 & 0.508 & 0.878 & 0.009 & 0.005 & 0.016 \\
\hline Anthracene & 0.018 & 0.012 & 0.026 & 0.014 & 0.011 & 0.019 & 0.009 & 0.003 & 0.030 & 0.001 & 0.001 & 0.001 \\
\hline Fluoranthene & 0.029 & 0.024 & 0.036 & 0.019 & 0.011 & 0.039 & 0.141 & 0.113 & 0.193 & 0.004 & 0.003 & 0.004 \\
\hline Pyrene & 0.040 & 0.031 & 0.048 & 0.027 & 0.021 & 0.036 & 0.081 & 0.055 & 0.100 & 0.003 & 0.002 & 0.004 \\
\hline Benzo(a)anthracene & 0.002 & $9.0 \mathrm{E}-06$ & 0.006 & n.d. & n.d. & n.d. & 0.006 & 0.003 & 0.010 & n.d. & n.d. & n.d. \\
\hline Chrysene & 0.007 & 0.003 & 0.014 & 0.003 & $1.00 \mathrm{E}-04$ & 0.007 & 0.052 & 0.025 & 0.084 & n.d. & n.d. & n.d. \\
\hline Benzo $(b+k) f l u o r a n t h e n e$ & 0.002 & 7.0E-06 & 0.006 & 0.001 & $8.00 \mathrm{E}-06$ & 0.003 & 0.050 & 0.015 & 0.104 & n.d. & n.d. & n.d. \\
\hline Benzo(a)pyrene & 0.001 & 7.0E-06 & 0.002 & $3.00 \mathrm{E}-04$ & $8.00 \mathrm{E}-06$ & 0.001 & 0.004 & 0.002 & 0.009 & n.d. & n.d. & n.d. \\
\hline Indeno(123-cd)pyrene & 0.002 & $7.4 \mathrm{E}-05$ & 0.004 & 0.001 & 7.00E-05 & 0.003 & 0.014 & 0.006 & 0.020 & n.d. & n.d. & n.d. \\
\hline Dibenzo(ah)anthracene & n.d. & n.d. & n.d. & n.d. & n.d. & n.d. & 0.004 & 0.002 & 0.007 & n.d. & n.d. & n.d. \\
\hline Benzo(ghi)perylene & 0.004 & 0.001 & 0.008 & 0.001 & $8.00 \mathrm{E}-05$ & 0.004 & 0.020 & 0.009 & 0.029 & n.d. & n.d. & n.d. \\
\hline
\end{tabular}

n.d.: below detection limits

n.q.: high blank contamination

${ }^{1)}$ Data acquired from http://ebas.nilu.no, last access: 20 June 2020

$270 \quad{ }^{2-4)}$ Volume sampled: $350-450 \mathrm{~m}^{3}, 650 \mathrm{~m}^{3}$, and $1300 \mathrm{~m}^{3}$, respectively

${ }^{5)}$ Possibly influenced by blank levels

NOTE the chemical profiles are presented as Figure S3 
Table S14: Comparison of average concentrations (G+P; $\mathrm{pg} \mathrm{m}^{-3}$ ) of PAHs, oxy-PAHs and nitro-PAHs measured in Longyearbyen with those previously reported in the literature for rural sites worldwide

\begin{tabular}{|c|c|c|c|c|c|c|}
\hline Region & Season & Sampling phase & Type of site & $\begin{array}{c}\text { Number of } \\
\text { compared } \\
\text { compounds* }\end{array}$ & $\begin{array}{r}\text { Average sum } \\
\text { concentration } \\
\left(\mathrm{pg} \mathrm{m}^{-3}\right)\end{array}$ & Reference \\
\hline \multicolumn{7}{|l|}{ Parent PAHs } \\
\hline France, Marseille & Summer 2004 & $\mathrm{G}+\mathrm{P}$ & Rural & 13 & 15959 & (Albinet et al., 2007) \\
\hline Denmark, Ris $\emptyset$ & Winter 1998 & $\mathrm{P}$ & Semi-rural & 9 & 4390 & (Feilberg et al., 2001) \\
\hline Eastern England, Weybourne & Winter 2010 & $\mathrm{G}+\mathrm{P}$ & Background & 12 & 2298 & (Alam et al., 2014) \\
\hline Southern China, Wanqingsha & Autumn 2010 & $\mathrm{G}+\mathrm{P}$ & Background & 15 & 88950 & (Huang et al., 2014b) \\
\hline Subarctic, Finland, Pallas & Autumns 1996-2015 & $\mathrm{G}+\mathrm{P}$ & Background & 12 & 403 & (Yu et al., 2019) \\
\hline Arctic, Canada, Alert & Autumns 1992-2015 & $\mathrm{G}+\mathrm{P}$ & Background & 15 & 81 & (Yu et al., 2019) \\
\hline Arctic, Svalbard, Zeppelin & Autumns 1994-2015 & $\mathrm{G}+\mathrm{P}$ & Background, altitude & 13 & 185 & (Yu et al., 2019) \\
\hline Southern Norway, Birkenes & Autumn 2018 & $\mathrm{G}+\mathrm{P}$ & Background & 15 & 1480 & (NILU, 2018) \\
\hline Arctic, Svalbard, Zeppelin & Autumn 2018 & $\mathrm{G}+\mathrm{P}$ & Background, altitude & 15 & 28 & (NILU, 2018) \\
\hline Arctic, Svalbard, UNIS & Autumn 2018 & $\mathrm{G}+\mathrm{P}$ & Semi-urban & 15 & 749 & This study \\
\hline Arctic, Svalbard, Adventdalen & Autumn 2018 & $\mathrm{G}+\mathrm{P}$ & Rural & 15 & 369 & This study \\
\hline \multicolumn{7}{|l|}{ Nitro-PAHs } \\
\hline Northern China & Annual 2010-2011 & $\mathrm{G}+\mathrm{P}$ & Rural & 12 & ${ }^{* *} 911$ & (Li et al., 2015) \\
\hline Southern China, Wanqingsha & Autumn 2010 & $\mathrm{G}+\mathrm{P}$ & Rural & 23 & 4021 & (Huang et al., 2014b) \\
\hline France, Marseille & Summer 2004 & $\mathrm{G}+\mathrm{P}$ & Rural & 9 & 26 & (Albinet et al., 2007) \\
\hline France, The Alps, Chamonix & Winter 2002 & $\mathrm{P}$ & Rural, altitude & 10 & 9 & (Albinet et al., 2008a) \\
\hline France, The Alps, Maurienne & Winter 2002 & $\mathrm{G}+\mathrm{P}$ & Rural & 15 & ${ }^{* *} 352$ & (Albinet et al., 2008a) \\
\hline Danmark, Ris $\varnothing$ & Winter 1998 & $\mathrm{P}$ & Semi-rural & 4 & 152 & (Feilberg et al., 2001) \\
\hline Central Europe, Košetice & Winter 2017 & $\mathrm{G}+\mathrm{P}$ & Background & 8 & 15 & (Lammel et al., 2020) \\
\hline Southern Sweden, Råö & Spring 2008 & $\mathrm{G}+\mathrm{P}$ & Background & 8 & 40 & (Brorström-Lundén et al., 2010) \\
\hline Subarctic Finland, Pallas & Spring 2008 & $\mathrm{G}+\mathrm{P}$ & Background & 8 & 19 & (Brorström-Lundén et al., 2010) \\
\hline Arctic, Svalbard, UNIS & Autumn 2018 & $\mathrm{G}+\mathrm{P}$ & Semi-urban & 11 & 37 & This study \\
\hline Arctic, Svalbard, Adventdalen & Autumn 2018 & $\mathrm{G}+\mathrm{P}$ & Rural & 11 & 27 & This study \\
\hline \multicolumn{7}{|l|}{ Oxy-PAHs } \\
\hline Northern China & Annual 2010-2011 & $\mathrm{G}+\mathrm{P}$ & Rural & 3 & ${ }^{* *} 10552$ & (Li et al., 2015) \\
\hline Southern China, Wanqingsha & Autumn 2010 & $\mathrm{G}+\mathrm{P}$ & Rural & 5 & 12940 & (Huang et al., 2014b) \\
\hline France, Marseille & Summer 2004 & $\mathrm{G}+\mathrm{P}$ & Rural & 4 & 1093 & (Albinet et al., 2007) \\
\hline France, The Alps, Chamonix & Winter 2002 & $\mathrm{P}$ & Rural, altitude & 5 & 790 & (Albinet et al., 2008a) \\
\hline France, The Alps, Maurienne & Winter 2002 & $\mathrm{G}+\mathrm{P}$ & Rural & 5 & ${ }^{* *} 6875$ & (Albinet et al., 2008a) \\
\hline Central Europe, Košetice & Winter 2017 & $\mathrm{G}+\mathrm{P}$ & Rural & 5 & 336 & (Lammel et al., 2020) \\
\hline Eastern England, Weybourne & Winter 2010 & $\mathrm{G}+\mathrm{P}$ & Rural & 4 & 591 & (Alam et al., 2014) \\
\hline Southern Sweden, Råö & Spring 2008 & $\mathrm{G}+\mathrm{P}$ & Background & 4 & 583 & (Brorström-Lundén et al., 2010) \\
\hline Subarctic, Finland, Pallas & Spring 2008 & $\mathrm{G}+\mathrm{P}$ & Background & 4 & 239 & (Brorström-Lundén et al., 2010) \\
\hline Arctic, Svalbard, UNIS & Autumn 2018 & $\mathrm{G}+\mathrm{P}$ & Semi-urban & 6 & 471 & This study \\
\hline Arctic, Svalbard, Adventdalen & Autumn 2018 & $\mathrm{G}+\mathrm{P}$ & Rural & 6 & 233 & This study \\
\hline
\end{tabular}

*within similar detectable compounds

**average of sites within type of site 
275 Table S15. Spearman correlation of selected PAH \% PMs with ambient temperature and specific humidity for Adventdalen data $(n=6)$

\begin{tabular}{lrr} 
& Temperature & Humidity \\
\hline \%PM Fluorene & -0.030 & -0.030 \\
\%PM Phenanthrene & 0.131 & 0.131 \\
\%PM Anthracene & -0.393 & -0.393 \\
\%PM Fluoranthene & -0.371 & -0.371 \\
\%PM Pyrene & -0.429 & -0.429 \\
\%PM Chrysene & $\mathbf{- 0 . 7 0 0}$ & $\mathbf{- 0 . 7 0 0}$ \\
\%PM 1-Nitronaphthale & -0.655 & -0.655 \\
\%PM 2-Nitronaphthale & -0.698 & -0.698 \\
\%PM 2-Nitrobiphenyl & -0.455 & -0.455 \\
\%PM 9-Fluorenone & -0.516 & -0.516 \\
\%PM 9,10-Anthraquinone & -0.205 & -0.205 \\
\%PM cPPhen-4 & $\mathbf{- 0 . 7 5 9}$ & $\mathbf{- 0 . 7 5 9}$ \\
\%PM 2+3-Nitrofluoranthene & $\mathbf{- 0 . 8 7 2}$ & $\mathbf{- 0 . 8 7 2}$ \\
\%PM 2-NFlu & -0.030 & -0.030 \\
\%PM 9-NAnt & 0.900 & 0.900 \\
\hline
\end{tabular}

Table S16. Spearman correlation of selected PAH concentrations $(G+P)$ with precipitation for UNIS data $(\mathbf{n}=6)$

\begin{tabular}{lr} 
& Precipitation \\
\hline Pyrene & -0.829 \\
Phenanthrene & $-0,543$ \\
Fluoranthene & $-0,543$ \\
Chrysene & -0.943 \\
Benzo(b+k)fluoranthene & -0.886 \\
Indeno(1,2,3-cd)pyrene & -0.812 \\
Benzo(g,h,i)perylene & -0.886 \\
Benzo(a)fluoren-11-one & -0.714 \\
Benzo(a)anthracene-7,12-dione & -0.829 \\
\hline
\end{tabular}


Table S17. Spearman correlation of PAH concentrations $(G+P)$ with weather parameters for Adventdalen data $(\mathbf{n}=6)$

\begin{tabular}{lrrr} 
& Solar radiation & Temperature & Humidity \\
\hline Acenaphtylene & $\mathbf{- 0 . 8 2 9}$ & $\mathbf{- 1 . 0 0 0}$ & $\mathbf{- 1 . 0 0 0}$ \\
Acenaphthene & -0.600 & -0.771 & -0.771 \\
Fluorene & -0.486 & -0.714 & -0.714 \\
Phenanthrene & -0.371 & -0.657 & -0.657 \\
Anthracene & -0.543 & -0.714 & -0.714 \\
Fluoranthene & $\mathbf{- 0 . 9 4 3}$ & -0.771 & -0.771 \\
Pyrene & $\mathbf{- 0 . 8 8 6}$ & $\mathbf{- 0 . 8 8 6}$ & $\mathbf{- 0 . 8 8 6}$ \\
Chrysene & -0.600 & -0.771 & -0.771 \\
1-Nitronaphthale & -0.771 & $\mathbf{- 0 . 9 4 3}$ & $\mathbf{- 0 . 9 4 3}$ \\
2-Nitronaphthale & -0.257 & -0.429 & -0.429 \\
9-Fluorenone & -0.543 & $\mathbf{- 0 . 8 2 9}$ & $\mathbf{- 0 . 8 2 9}$ \\
9,10-Anthraquinone & 0.086 & -0.314 & -0.314 \\
cPPen-4 & -0.771 & -0.771 & -0.771 \\
2-Nitrofluorene & 0.200 & 0.086 & 0.086 \\
9-Nitroanthracene & 0.657 & 0.714 & 0.714 \\
Benzo(a)fluoren-11-one & -0.600 & -0.771 & -0.771 \\
2+3-Nitrofluoranthene & 0.657 & 0.657 & 0.657 \\
\hline & & & \\
\hline
\end{tabular}


Table S18. \%PM obtained in this study compared to literature data

\begin{tabular}{|c|c|c|c|c|c|}
\hline & $\begin{array}{r}\text { UNIS }^{1)} \\
\text { Sub-urban, } \\
\text { autumn }\end{array}$ & $\begin{array}{l}\text { Adventdalen }^{1)} \\
\text { Rural, autumn }\end{array}$ & $\begin{array}{r}\text { Southern China }^{2)} \\
\text { Rural, autumn }\end{array}$ & $\begin{array}{r}\text { Southeastern France }^{3)} \\
\text { Urban, annual }\end{array}$ & $\begin{array}{r}\text { The Apls, France }{ }^{4)} \\
\text { Rural, winter }\end{array}$ \\
\hline Acenaphtylene & $0 \pm 0.0$ & $0 \pm 0$ & 0.9 & - & - \\
\hline Acenaphthene & $0 \pm 0.0$ & $0 \pm 0$ & 1.9 & 5.9 & - \\
\hline Fluorene & $1.1 \pm 1.0$ & $1.8 \pm 3.0$ & 1.3 & 2.0 & - \\
\hline Phenanthrene & $6.5 \pm 8.6$ & $3.7 \pm 9.0$ & 0.6 & 3.0 & - \\
\hline Anthracene & $0 \pm 0.0$ & $3.5 \pm 8.5$ & 3.4 & 4.3 & - \\
\hline Fluoranthene & $40.5 \pm 19.4$ & $23.2 \pm 9.2$ & 11.0 & 15.7 & - \\
\hline Pyrene & $26.8 \pm 18.6$ & $15.8 \pm 11.0$ & 16.5 & 20.0 & - \\
\hline Benzo(a)anthracene & $68.2 \pm 2.4$ & - & 85.3 & 65.7 & - \\
\hline Chrysene & $81.7 \pm 10.3$ & $64.1 \pm 18.7$ & 85.3 & 80.7 & - \\
\hline Benzo(b+k)fluoranthene & $100 \pm 0.0$ & $100 \pm 0$ & 98.4 & 100.0 & - \\
\hline Benzo(a)pyrene & $100 \pm 0.0$ & $100 \pm 0$ & 98.8 & 100.0 & 100.0 \\
\hline Indeno(1,2,3-cd)pyrene & $100 \pm 0.0$ & $100 \pm 0$ & 99.6 & 98.1 & - \\
\hline Dibenzo(a,h)anthracene & - & - & 100.0 & 100.0 & - \\
\hline Benzo(g,h,i)perylene & $100 \pm 0.0$ & $100 \pm 0$ & 99.6 & 97.8 & - \\
\hline 1-Nitronaphthalene & $0.1 \pm 0.2$ & $1.5 \pm 3.6$ & 1.7 & 2.1 & 11.3 \\
\hline 2-Nitronaphthalene & $3.1 \pm 5.6$ & $5.4 \pm 8.0$ & 0.7 & 2.3 & 8.5 \\
\hline 2-Nitrobiphenyl & $10.1 \pm 8.3$ & $5.9 \pm 10.0$ & 14.5 & 0 & - \\
\hline 9-Fluorenone & $41.7 \pm 22.3$ & $25.5 \pm 13.4$ & 3.8 & 2.2 & 38.8 \\
\hline 4-Nitrobiphenyl & $0 \pm 0.0$ & - & 3.8 & - & - \\
\hline 1,5-Dinitronaphthalene & $80.0 \pm 44.7$ & $53.9 \pm 53.6$ & 39.3 & 100.0 & - \\
\hline 9,10 -Anthraquinone & $37.5 \pm 22.4$ & $43.9 \pm 1.4$ & 43.0 & 55.8 & 97.8 \\
\hline 5-Nitroacenaphthene & - & - & 95.0 & 53.3 & - \\
\hline cPPhen-4 & $65.5 \pm 10.6$ & $38.1 \pm 20.2$ & 20.4 & - & - \\
\hline 2-Nitrofluorene & $15.1 \pm 7.5$ & $4.2 \pm 9.5$ & - & 0.0 & 87.5 \\
\hline 9-Nitroanthracene & - & $57.8 \pm 29.7$ & 95.0 & 64.7 & 95.8 \\
\hline 9-Nitrophenanthrene & - & $25 \pm 50$ & - & 66.7 & 88.3 \\
\hline Benzo(a)fluoren-11-one & $100 \pm 0.0$ & $100 \pm 0$ & - & 95.5 & 99.8 \\
\hline Benzanthrone & $96.7 \pm 4.8$ & $100 \pm 0$ & 98.2 & 100.0 & 100.0 \\
\hline 2+3-Nitrofluoranthene & $94.5 \pm 13.5$ & $79.8 \pm 16.5$ & 97.0 & 98.6 & 99.0 \\
\hline $\begin{array}{l}\text { Benzo(a)anthracene- } \\
\text { 7,12-dione }\end{array}$ & $100 \pm 0.0$ & $100 \pm 0$ & 99.3 & 99.4 & 100.0 \\
\hline
\end{tabular}

${ }^{1)}$ This study; ${ }^{2)}$ (Huang et al., 2014a); ${ }^{3)}$ (Tomaz et al., 2016); ${ }^{4)}$ (Albinet et al., 2008b) 
Table S19. Combustion engine vehicles in Longyearbyen as of 2018

\begin{tabular}{lrrr} 
& Diesel engine & Gasoline engine & Total \\
\hline Passenger cars & 522 & 553 & 1114 \\
Vans & 276 & 17 & 293 \\
Lorry & 52 & 8 & 60 \\
Buses & 26 & - & 26 \\
Tractors & 33 & 32 & 65 \\
Recreational Boats & & & \\
& n.a. & n.a. & 718 \\
\hline
\end{tabular}

Data acquired from Statistics Norway (www.ssb.no), last access: 20 June 2020 n.a. this information is not available 
Table S20. Eigenanalysis of the correlation matrix and Eigenvectors for Adventdalen data

\begin{tabular}{lrr} 
Variable & PC1 & PC2 \\
\hline Eigenvalue & 8,7122 & 3,8497 \\
Proportion & $\mathbf{0 , 5 1 2}$ & $\mathbf{0 , 2 2 6}$ \\
Cumulative & 0,512 & 0,739 \\
\hline Flu/(Flu+Pyr) & $-0,002$ & $\mathbf{- 0 , 4 2 7}$ \\
IPyr/(IPyr+BPer) & $\mathbf{0 , 2 3 8}$ & 0,225 \\
Flu & 0,207 & $\mathbf{- 0 , 3 6 6}$ \\
Phe & $\mathbf{0 , 1 7 6}$ & $\mathbf{- 0 , 3 8 8}$ \\
Flt & $\mathbf{0 , 2 4 3}$ & 0,294 \\
Pyr & $\mathbf{0 , 3 0 0}$ & 0,225 \\
9-Flu & 0,170 & $\mathbf{- 0 , 2 8 7}$ \\
9,10-AntQ & 0,153 & $-0,090$ \\
cPPhe-4 & $\mathbf{0 , 3 1 9}$ & 0,096 \\
BaFlu-11 & $\mathbf{0 , 2 8 5}$ & 0,194 \\
BaAnt-7,12 & $\mathbf{0 , 2 4 0}$ & 0,047 \\
1-NNap & 0,253 & $\mathbf{- 0 , 3 0 8}$ \\
9-NAnt & $\mathbf{0 , 2 2 5}$ & 0,054 \\
2+3-NFlt & $\mathbf{- 0 , 2 4 5}$ & $-0,252$ \\
Temperature & $\mathbf{0 , 3 1 8}$ & 0,068 \\
Humidity & $\mathbf{- 0 , 2 8 7}$ & 0,187 \\
Solar Radiation & $\mathbf{- 0 , 2 6 4}$ & $-0,073$ \\
\hline & & \\
\hline
\end{tabular}

295 Table S21. Spearman correlation of PAH concentrations $(\mathbf{G + P}$; $\mathbf{n = 6})$ with diagnostic ratios for Adventdalen data

\begin{tabular}{lrr} 
& $\begin{array}{r}\text { IPyr/(IPyr+BPer) } \\
\text { traffic }\end{array}$ & $\begin{array}{r}\text { Flu/(Flu+Pyr) } \\
\text { coal burning }\end{array}$ \\
\hline Flu & 0,270 & $\mathbf{0 , 7 7 1}$ \\
Phe & 0,101 & $\mathbf{0 , 7 7 1}$ \\
Ant & $\mathbf{0 , 8 4 5}$ & 0,143 \\
Flt & 0,541 & $-0,257$ \\
Pyr & 0,439 & 0,257 \\
Chry & $\mathbf{0 , 7 7 8}$ & $-0,029$ \\
1-NNap & 0,439 & 0,429 \\
9-Flu & $-0,034$ & 0,314 \\
9,10-AntQ & $-0,101$ & 0,257 \\
cPPhe-4 & $\mathbf{0 , 7 7 8}$ & $-0,314$ \\
9-NAnt & $-0,135$ & $-0,371$ \\
9-NPhe & $\mathbf{0 , 7 8 9}$ & $-0,232$ \\
BaFlu-11 & $\mathbf{0 , 7 7 8}$ & $-0,029$ \\
2+3-NFlt & $-0,778$ & 0,086 \\
BaAnt-7,12 & $\mathbf{0 , 8 4 5}$ & 0,143 \\
\hline
\end{tabular}


Table S22. Extractions from certificate of quality: unleaded gasoline, RON95, Norway, summer

\begin{tabular}{|c|c|c|c|}
\hline Property & Units & Spec & Results \\
\hline Appearance & - & Clear \% Bright & Clear \& Bright \\
\hline Density at 15 deg. $C$ & $\mathrm{~kg} / \mathrm{m} 3$ & $720-775$ & 723.0 \\
\hline Reid Vapour Pressure & $\mathrm{kPa}$ & $\leq 67.0$ & 66.9 \\
\hline Colour & - & Undyed & Undyed \\
\hline Odour & - & & Merchantable \\
\hline Total Sulphur & $\mathrm{mg} / \mathrm{kg}$ & $\leq 10.0$ & 3.9 \\
\hline Copper Strip Corrosion $3 \mathrm{~h}$ at $50 \mathrm{deg}$. C & - & No.1 & No.1 \\
\hline Existent Gum & $\mathrm{mg} / 100 \mathrm{ml}$ & $\leq 5$ & $\leq 5$ \\
\hline Oxidation Stability & $\min$ & $\geq \overline{360}$ & $\geq 360$ \\
\hline MTBE & $\%$-vol & $\leq 0.5$ & $<0.1$ \\
\hline Oxygen content & $\%$-wt & $\leq 0.5$ & $<0.1$ \\
\hline Other Oxygenates & & Not allowed & Not added \\
\hline Lead & mg_Pb/1 & $\leq 5$ & $\leq 3$ \\
\hline Manganese & $\mathrm{mg} / \mathrm{l}$ & $\leq 2$ & $\leq 2$ \\
\hline Evaporated at $70 \mathrm{deg} . \mathrm{C}$ & $\%$-vol & $22.0-48.0$ & 37.4 \\
\hline Evaporated at $100 \mathrm{deg} . \mathrm{C}$ & $\%$-vol & $46.0-71.0$ & 60.9 \\
\hline Evaporated at $150 \mathrm{deg} . \mathrm{C}$ & $\%$-vol & $\geq 75.0$ & 90.8 \\
\hline Final Boiling Point & deg. C & $\leq 210.0$ & 195.0 \\
\hline Residue & $\%$-vol & $\leq 2.0$ & 1.0 \\
\hline Olefins & $\%$-vol & $\leq 18.0$ & 17.5 \\
\hline Aromatics & $\%$-vol & $\leq 35.0$ & 19.7 \\
\hline Benzene & $\%$-vol & $\leq 1.00$ & 0.84 \\
\hline Motor Octane Number & - & $\geq 85.0$ & 85.0 \\
\hline Research Octane Number & - & $\geq 95.0$ & 95.0 \\
\hline Silver strip corrosion ( $3 \mathrm{~h}$ at $50 \mathrm{deg}$. C) & - & $\leq 1$ & 0 \\
\hline
\end{tabular}

Table S23. Extractions from certificate of quality: B-base automotive diesel, CFPP-12, Norway

\begin{tabular}{|c|c|c|c|}
\hline Property & Units & Spec & Results \\
\hline Appearance at $20 \mathrm{deg} . \mathrm{C}$ & - & Clear \& Bright & Clear \& Bright \\
\hline Water & ppm-wt & $\leq 100$ & $\leq 100$ \\
\hline Density at 15 deg. $C$ & $\mathrm{~kg} / \mathrm{m} 3$ & $820.0-842.0$ & 840.8 \\
\hline Recovered at $250 \mathrm{deg} . \mathrm{C}$ & $\%$-vol & $\leq 64$ & 40 \\
\hline Recovered at $350 \mathrm{deg} . \mathrm{C}$ & $\%$-vol & $\geq 85$ & 98 \\
\hline $95 \%$ Recovered & deg. $\mathrm{C}$ & $\leq 360$ & 332 \\
\hline Flash Point & deg. C & $\geq 58$ & 62 \\
\hline Total Sulphur & $\mathrm{mg} / \mathrm{kg}$ & $\leq 10.0$ & 9.8 \\
\hline Copper Strip Corrosion $3 \mathrm{~h}$ at $50 \mathrm{deg}$. C & - & No. 1 & No. 1 \\
\hline Oxidation Stability & $\mathrm{g} / \mathrm{m} 3$ & $\leq 25$ & $\leq 25$ \\
\hline Viscosity at 40 deg. C & $\mathrm{mm} 2 / \mathrm{s}$ & $2.00-4.00$ & 2.57 \\
\hline Lubricity at $60 \mathrm{deg}$. C & um & $\leq 460$ & $\leq 460$ \\
\hline Cloud Point & deg. C & $\leq-1$ & -17 \\
\hline Cold Filter Plugging Point & deg. C & $\leq-12$ & -17 \\
\hline Carbon Residue on $10 \%$ Residue & $\%$-wt & $\leq 0.30$ & $\leq 0.30$ \\
\hline Ash & $\%$-wt & $\leq 0.01$ & $\leq 0.01$ \\
\hline Manganese & $\mathrm{mg} / \mathrm{l}$ & $\leq 2.0$ & $\leq 2.0$ \\
\hline Particulate Matter & $\mathrm{mg} / \mathrm{kg}$ & $\leq 24$ & $\leq 24$ \\
\hline Fatty acid methyl ester & $\%$-vol & $\leq 0.2$ & $\leq 0.2$ \\
\hline PAH & $\%$-wt & $\leq 8.0$ & 3.4 \\
\hline Cetane Index, four variable & - & $\geq 46.0$ & 48.8 \\
\hline Cetane Number & - & $\geq 51.0$ & 51.6 \\
\hline Aromatics, \%-wt & $\%$-wt & Report & 26.4 \\
\hline Conductivity & $\mathrm{pS} / \mathrm{m}$ & $>=50$ & 218 \\
\hline at temperature & deg. C & Report & 22 \\
\hline Stadis 425 & $\mathrm{mg} / \mathrm{l}$ & Report & 2.0 \\
\hline
\end{tabular}


Table S24. Ratios of nitro- and oxy-PAH to corresponding parent PAH at three locations

\begin{tabular}{|c|c|c|c|c|c|c|c|c|c|}
\hline & \multicolumn{3}{|c|}{ Power plant $(n=6)$} & \multicolumn{3}{|c|}{ UNIS $(n=6)$} & \multicolumn{3}{|c|}{ Adventdalen $(n=6)$} \\
\hline & Mean & STD & Median & Mean & STD & Median & Mean & STD & Median \\
\hline 9,10-AntQ/Ant & 12.16 & 6.53 & 10.47 & 9.44 & 3.80 & 7.63 & 4.02 & 2.40 & 4.57 \\
\hline 9-NAnt/Ant & 0.055 & 0.020 & 0.056 & 0.039 & 0.026 & 0.042 & 0.189 & 0.171 & 0.109 \\
\hline 9-FluQ/Flu & 1.67 & 0.29 & 1.72 & 1.65 & 0.95 & 1.30 & 2.35 & 0.79 & 2.15 \\
\hline BaFlu-11/Chry & 0.65 & 0.34 & 0.58 & 0.87 & 0.26 & 0.82 & 0.67 & 0.15 & 0.63 \\
\hline cPPhen-4/Pyr & 0.31 & 0.10 & 0.30 & 0.64 & 0.30 & 0.56 & 0.48 & 0.06 & 0.48 \\
\hline 9-FluQ/Phe & 0.47 & 0.13 & 0.42 & 0.67 & 0.38 & 0.51 & 0.55 & 0.09 & 0.56 \\
\hline $2+3-\mathrm{NFlt} / \mathrm{Flt}$ & 0.029 & 0.023 & 0.017 & 0.335 & 0.043 & 0.324 & 0.603 & 0.326 & 0.585 \\
\hline
\end{tabular}

Table S25. Spearman correlation of nitro- and oxy-PAH to corresponding parent PAH ratios with weather parameters in Adventdalen $(n=6)$

\begin{tabular}{lrrrr} 
& Solar radiation & Temperature & Humidity & Pressure \\
\hline 9-Flu/Flu & 0.029 & 0.029 & 0.029 & -0.029 \\
9,10-AntQ/Ant & 0.486 & -0.029 & -0.029 & 0.429 \\
9-FluQ/Phe & -0.429 & -0.486 & -0.486 & -0.257 \\
9-NAnt/Ant & 0.600 & $\mathbf{0 . 8 2 9}$ & $\mathbf{0 . 8 2 9}$ & 0.543 \\
2+3-NFlt/Flt & $\mathbf{0 . 8 8 6}$ & $\mathbf{0 . 7 1 4}$ & $\mathbf{0 . 8 2 9}$ & $\mathbf{0 . 9 4 3}$ \\
cPPhen-4/Pyr & 0.257 & 0.257 & 0.257 & -0.029 \\
\hline
\end{tabular}


Table S26. Spearman correlation of concentrations (G+P) of PAHs, nitro- and oxy-PAHs with each other for Adventdalen data (n=6)

\begin{tabular}{|c|c|c|c|c|c|c|c|c|c|c|c|c|c|c|c|c|c|c|}
\hline & Acy & Ace & Flu & Phe & Ant & Flt & Pyr & Chry & 1-Nnap & 2-Nnap & 9-Flu & 9,10-AntQ & cPPhen-4 & 2-Nflu & 9-Nant & 9-NPhe & BaFlu-11 & 2+3-NFlt \\
\hline Acy & & & & & & & & & & & & & & & & & & \\
\hline Ace & 0.771 & & & & & & & & & & & & & & & & & \\
\hline Flu & 0.714 & 0.600 & & & & & & & & & & & & & & & & \\
\hline Phe & 0.657 & 0.314 & 0.829 & & & & & & & & & & & & & & & \\
\hline Ant & 0.714 & 0.943 & 0.714 & 0.486 & & & & & & & & & & & & & & \\
\hline Flt & 0.771 & 0.714 & 0.314 & 0.143 & 0.600 & & & & & & & & & & & & & \\
\hline Pyr & 0.886 & 0.600 & 0.657 & 0.714 & 0.657 & 0.771 & & & & & & & & & & & & \\
\hline Chry & 0.771 & 1.000 & 0.600 & 0.314 & 0.943 & 0.714 & 0.600 & & & & & & & & & & & \\
\hline 1-Nnap & 0.943 & 0.714 & 0.829 & 0.829 & 0.771 & 0.657 & 0.943 & 0.714 & & & & & & & & & & \\
\hline 2-Nnap & 0.429 & 0.029 & 0.714 & 0.943 & 0.257 & -0.029 & 0.600 & 0.029 & 0.657 & & & & & & & & & \\
\hline 9-Flu & 0.829 & 0.371 & 0.486 & 0.714 & 0.314 & 0.429 & 0.714 & 0.371 & 0.771 & 0.543 & & & & & & & & \\
\hline 9,10-AntQ & 0.314 & 0.429 & 0.371 & 0.086 & 0.257 & -0.029 & -0.143 & 0.429 & 0.143 & -0.143 & 0.257 & & & & & & & \\
\hline cPPhen-4 & 0.771 & 0.829 & 0.314 & 0.257 & 0.771 & 0.886 & 0.771 & 0.829 & 0.714 & 0.029 & 0.486 & -0.029 & & & & & & \\
\hline 2-Nflu & -0.086 & -0.143 & 0.600 & 0.429 & 0.029 & -0.429 & -0.086 & -0.143 & 0.086 & 0.543 & -0.143 & 0.257 & -0.543 & & & & & \\
\hline 9-NPhe & 0.812 & 0.899 & 0.406 & 0.319 & 0.841 & 0.841 & 0.754 & 0.899 & 0.754 & 0.058 & 0.522 & 0.116 & 0.986 & -0.464 & -0.435 & & & \\
\hline BaFlu-11 & 0.771 & 1.000 & 0.600 & 0.314 & 0.943 & 0.714 & 0.600 & 1.000 & 0.714 & 0.029 & 0.371 & 0.429 & 0.829 & -0.143 & -0.657 & 0.899 & & \\
\hline 2+3-NFlt & -0.657 & -0.943 & -0.543 & -0.143 & -0.886 & -0.771 & -0.543 & -0.943 & -0.600 & 0.086 & -0.143 & -0.314 & -0.771 & 0.086 & 0.714 & -0.812 & -0.943 & \\
\hline BaAnt- 7,12 & 0.714 & 0.943 & 0.714 & 0.486 & 1.000 & 0.600 & 0.657 & 0.943 & 0.771 & 0.257 & 0.314 & 0.257 & 0.771 & 0.029 & -0.543 & 0.841 & 0.943 & -0.886 \\
\hline
\end{tabular}


Table S27. Eigenanalysis of the correlation matrix and Eigenvectors for UNIS data

\begin{tabular}{lrr} 
Variable & PC1 & PC2 \\
\hline Eigenvalue & 8,9948 & 3,5126 \\
Proportion & $\mathbf{0 , 5 2 9}$ & $\mathbf{0 , 2 0 7}$ \\
Cumulative & 0,529 & 0,736 \\
\hline BbkFlt/BPer & $\mathbf{0 , 2 3 5}$ & $-0,066$ \\
Flu/(Flu+Pyr) & $-0,195$ & $\mathbf{- 0 , 3 2 6}$ \\
Flt/(Flt+ Pyr) & $-0,062$ & $\mathbf{0 , 4 5 8}$ \\
Phe & $-0,117$ & $\mathbf{- 0 , 4 2 6}$ \\
Flt & $\mathbf{0 , 2 9 4}$ & 0,162 \\
Pyr & $\mathbf{0 , 3 0 8}$ & $-0,110$ \\
Chry & $\mathbf{0 , 2 8 9}$ & $-0,217$ \\
BaPyr & $\mathbf{0 , 3 2 3}$ & 0,071 \\
BPer & $\mathbf{0 , 2 6 6}$ & $-0,284$ \\
1-NNap & $\mathbf{0 , 1 9 3}$ & $-0,057$ \\
2-NNap & $\mathbf{0 , 3 0 3}$ & 0,119 \\
9-Flu & $-0,093$ & $\mathbf{0 , 2 7 4}$ \\
9,10-AntQ & $-0,182$ & $\mathbf{- 0 , 3 7 4}$ \\
cPPeh-4 & 0,076 & $\mathbf{0 , 2 0 3}$ \\
BaFlu-11 & $\mathbf{0 , 3 2 0}$ & $-0,056$ \\
BZT & $\mathbf{0 , 2 8 2}$ & 0,100 \\
BaAnt-7,12 & $\mathbf{0 , 3 0 2}$ & $-0,203$ \\
\hline & & \\
\hline & &
\end{tabular}

Table S28. Spearman correlation of PAH concentrations $(\mathrm{G}+\mathrm{P} ; \mathrm{n}=6)$ with diagnostic ratios for UNIS data

\begin{tabular}{lrrrr} 
& IPyr/(IPyr+BPer) & $\begin{array}{r}\text { BbkFlt/BPer } \\
\text { traffic }\end{array}$ & $\begin{array}{r}\text { Flu/(Flu+Pyr) } \\
\text { diesel/gasoline }\end{array}$ & $\begin{array}{r}\text { Flt/(Flt+Pyr) } \\
\text { coal combustion }\end{array}$ \\
\hline Flu & $-0,429$ & 0,143 & $\mathbf{0 , 7 1 4}$ & $-0,714$ \\
Phe & 0,086 & 0,200 & $\mathbf{0 , 5 4 3}$ & $-0,200$ \\
Flt & 0,886 & $\mathbf{0 , 7 7 1}$ & $-0,829$ & 0,143 \\
Pyr & 0,771 & $\mathbf{0 , 8 8 6}$ & $-0,714$ & $-0,143$ \\
Chry & 0,543 & $\mathbf{0 , 8 8 6}$ & $-0,371$ & $-0,486$ \\
BbkFlt & 0,600 & $\mathbf{0 , 9 4 3}$ & $-0,200$ & $-0,314$ \\
BaPyr & 0,759 & $\mathbf{0 , 8 8 0}$ & $-0,698$ & $-0,030$ \\
IPyr & 0,754 & $\mathbf{0 , 9 8 6}$ & $-0,464$ & $-0,232$ \\
BPer & 0,600 & $\mathbf{0 , 9 4 3}$ & $-0,200$ & $-0,314$ \\
1-NNap & $\mathbf{0 , 5 4 3}$ & 0,429 & $-0,600$ & $-0,086$ \\
2-NNap & $\mathbf{0 , 7 7 1}$ & 0,657 & $-0,943$ & 0,086 \\
9-Flu & 0,371 & 0,257 & 0,029 & $\mathbf{0 , 6 5 7}$ \\
9,10-AntQ & $-0,829$ & $-0,600$ & $\mathbf{0 , 6 5 7}$ & $-0,486$ \\
cPPeh-4 & 0,086 & 0,314 & 0,314 & 0,200 \\
BaFlu-11 & $\mathbf{0 , 7 7 1}$ & $\mathbf{0 , 8 8 6}$ & $-0,714$ & $-0,143$ \\
BZT & $\mathbf{0 , 7 5 4}$ & 0,638 & $-0,696$ & 0,087 \\
BaAnt-7,12 & $\mathbf{0 , 7 1 4}$ & $\mathbf{0 , 9 4 3}$ & $-0,543$ & $-0,314$ \\
\hline & & & & \\
\hline
\end{tabular}


Figure S1. Wind rose diagrams for UNIS (samples U1-U7) and Adventdalen (samples A1-A7) sampling stations
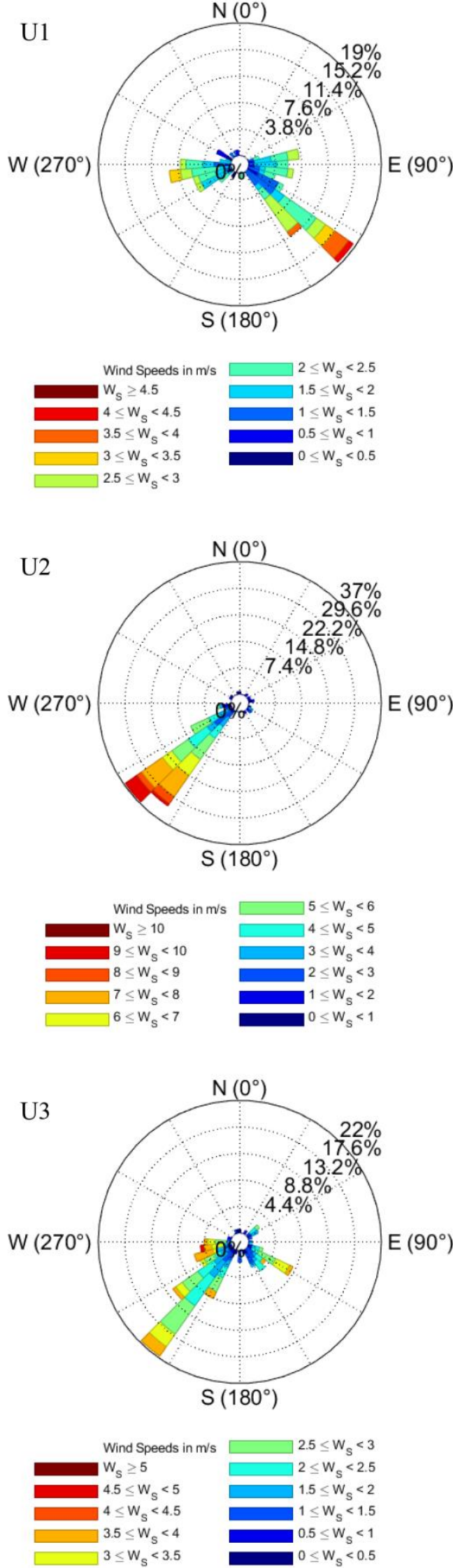
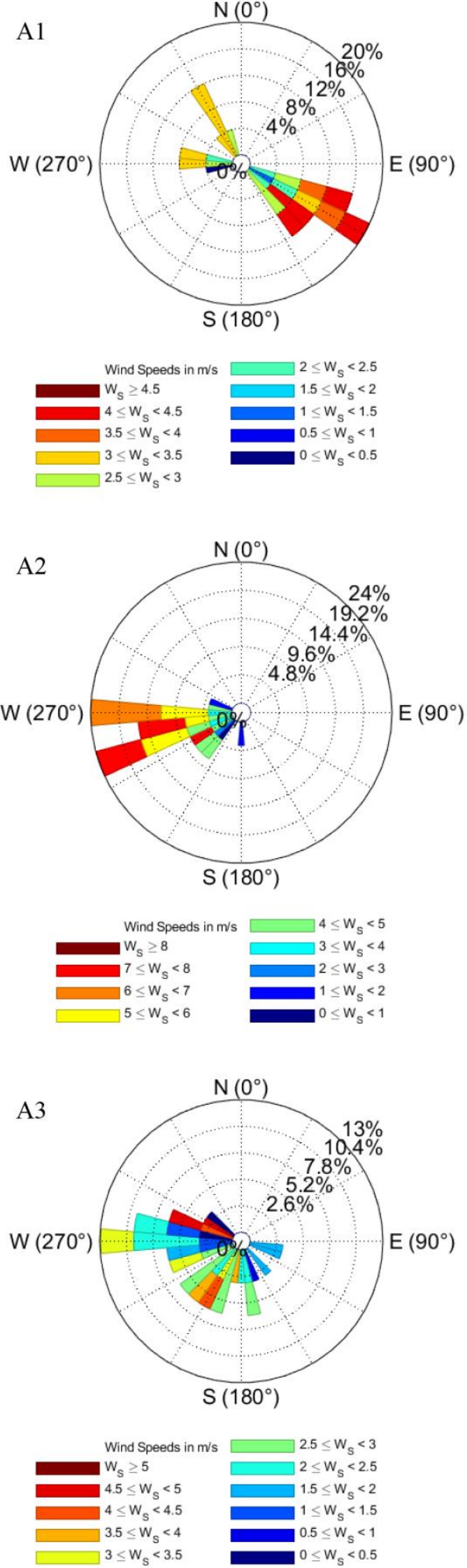
Figure S1 continued
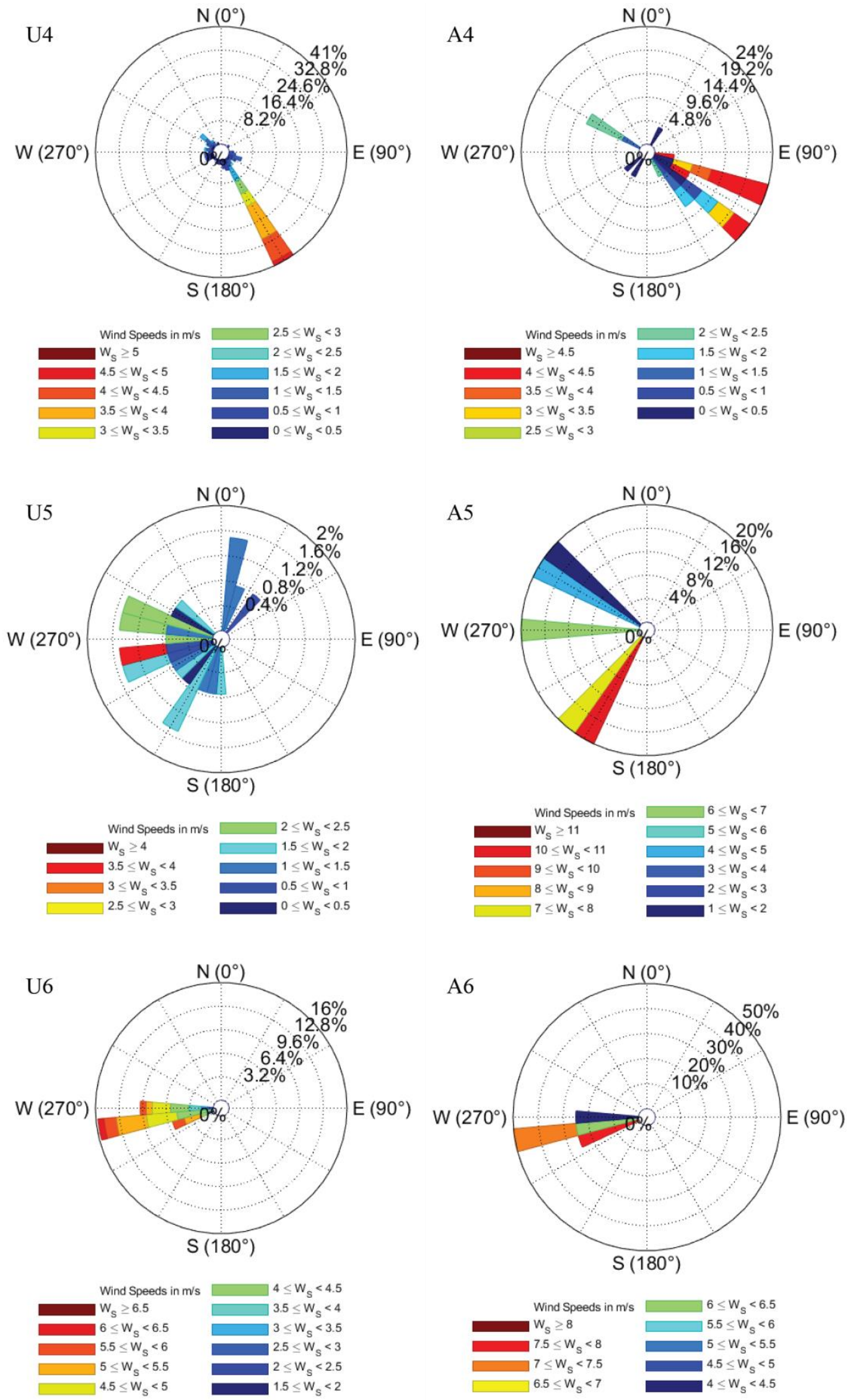
Figure S1 continued
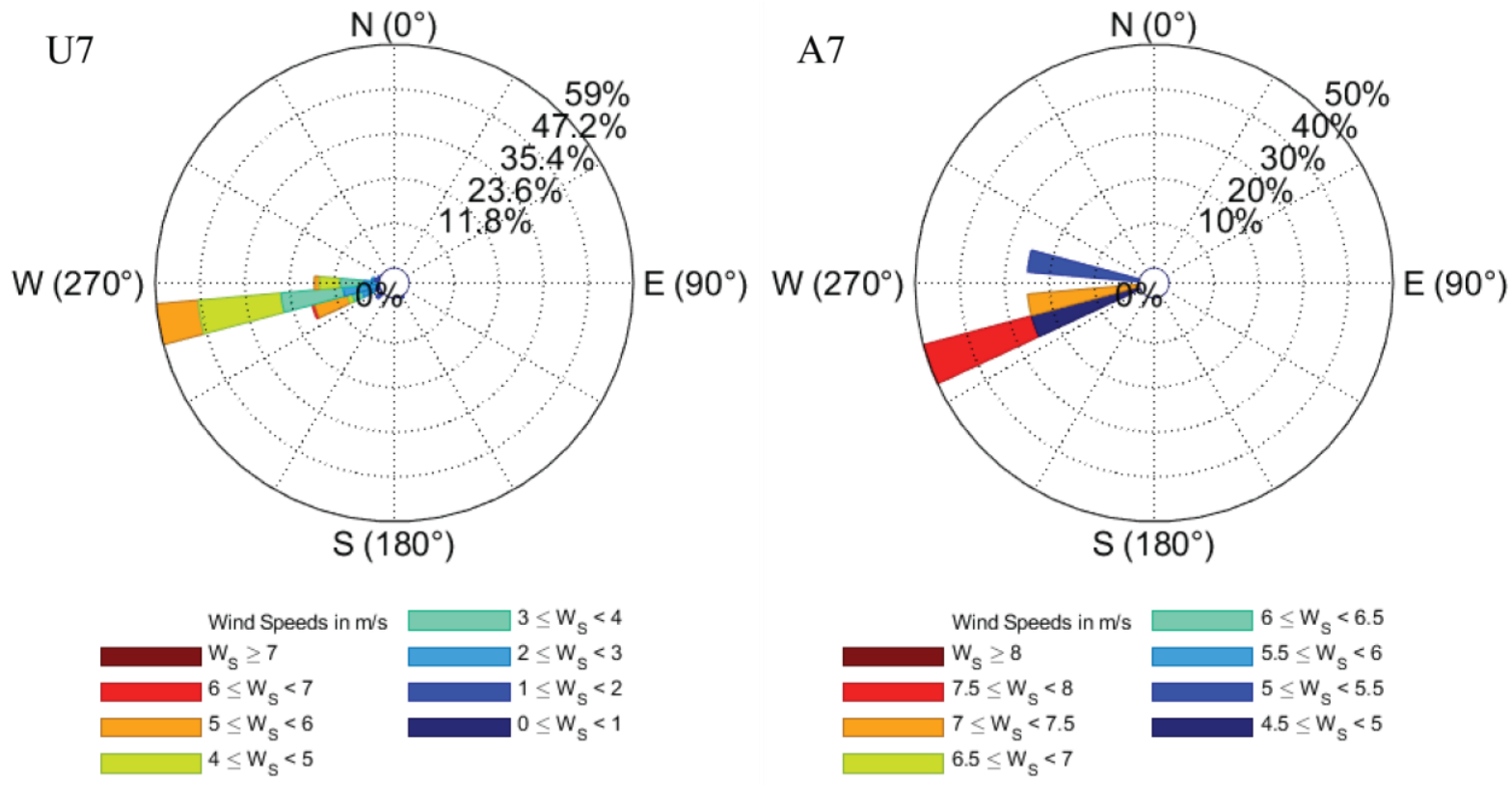

Figure S2. UNIS and Adventdalen chemical profiles of (a) PAHs, (b) oxy-PAHs, and (c) nitro-PAHs

(a) $100 \%$

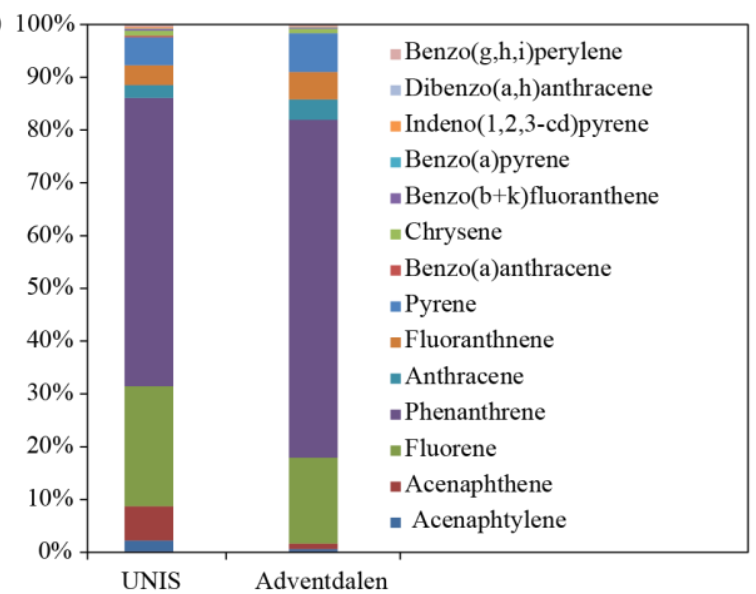

(c) $100 \%$

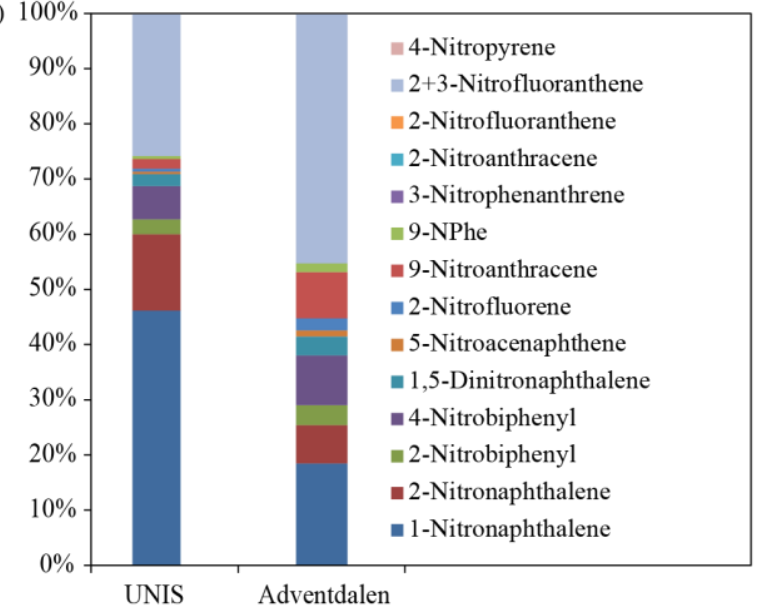

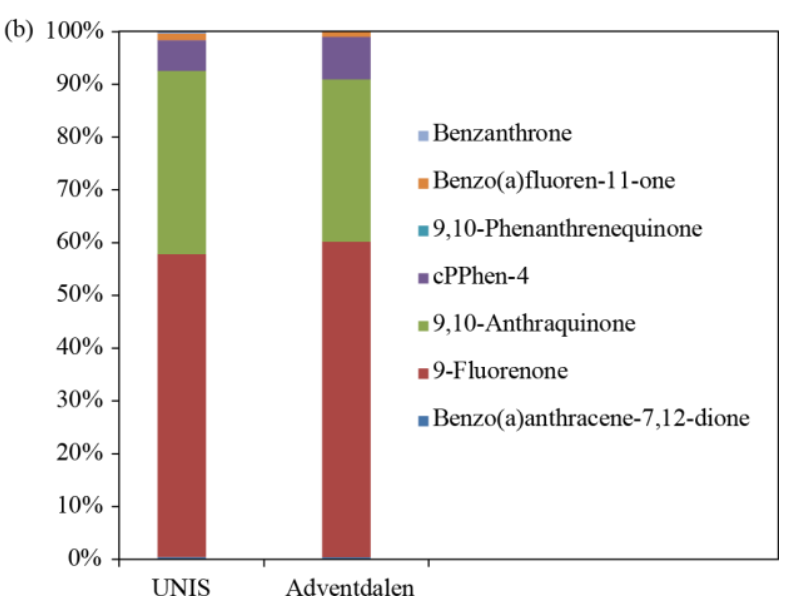


Figure S3. The 15 PAH profiles for different stations in Svalbard (UNIS, Adventdalen, Zeppelin) and the mainland Norway (Birkenes) measured in autumn 2018*

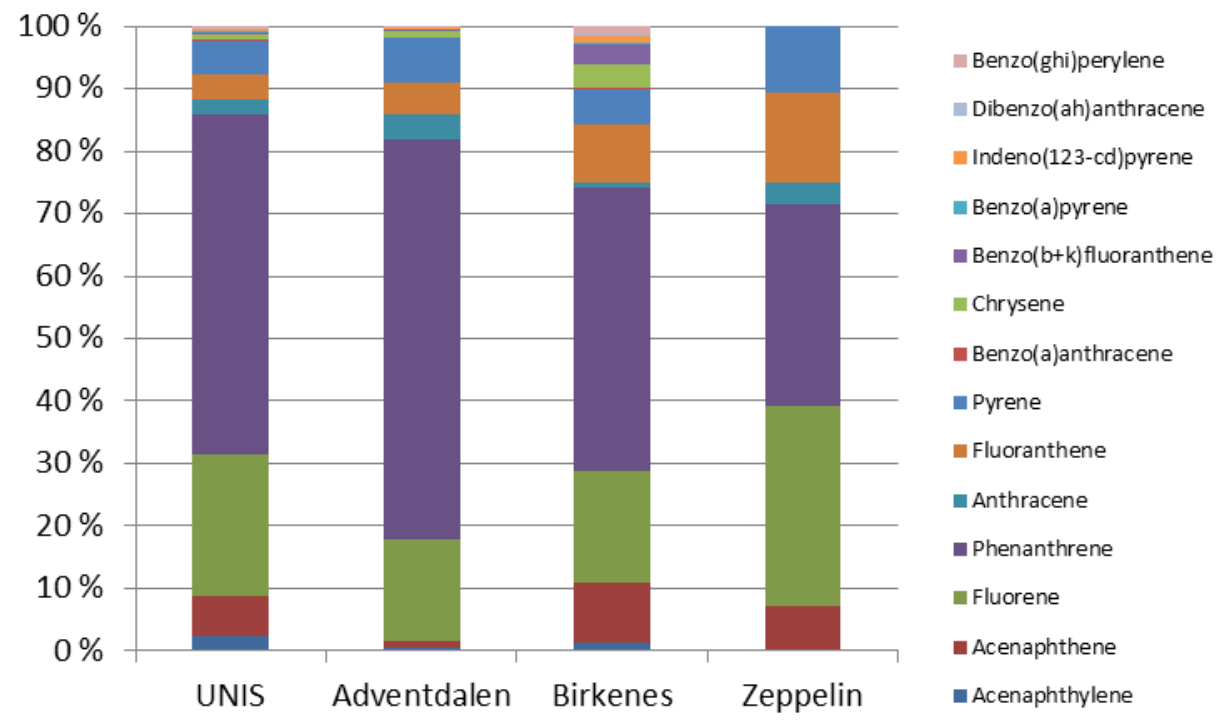

* Based on Table S13 data

Figure S4. 5-day back trajectories of Longyearbyen*

NOAA HYSPLIT MODEL

Backward trajectories ending at 0900 UTC 29 Aug 18 GDAS Meteorological Data

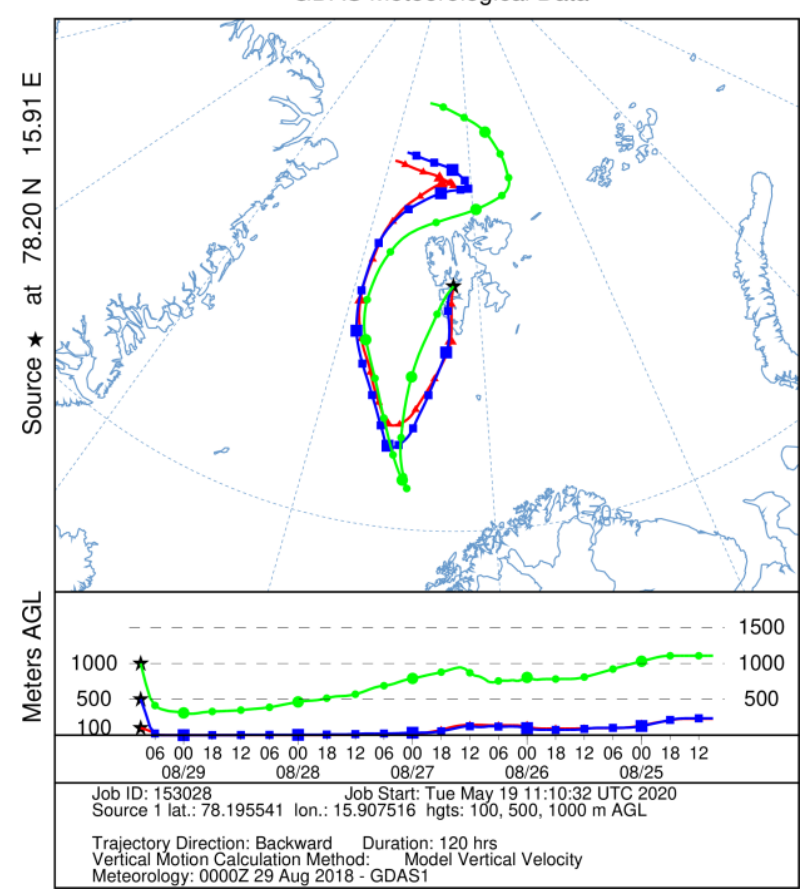

NOAA HYSPLIT MODEL

Backward trajectories ending at 2100 UTC 31 Aug 18 GDAS Meteorological Data

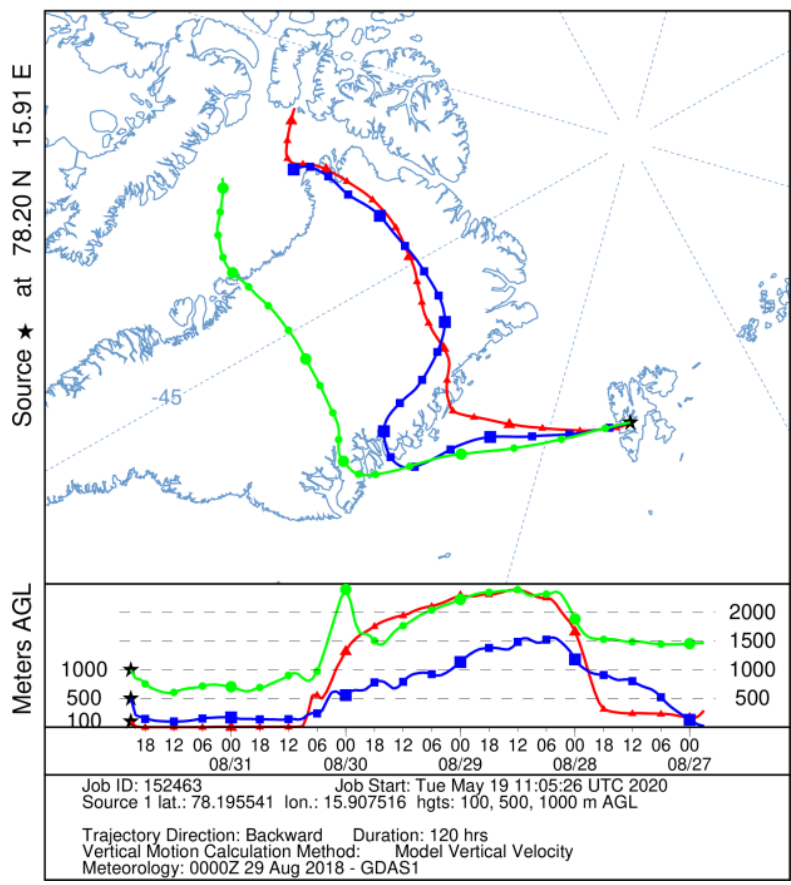


Figure $\mathbf{S 4}$ continued

NOAA HYSPLIT MODEL

Backward trajectories ending at 1900 UTC 07 Sep 18 GDAS Meteorological Data

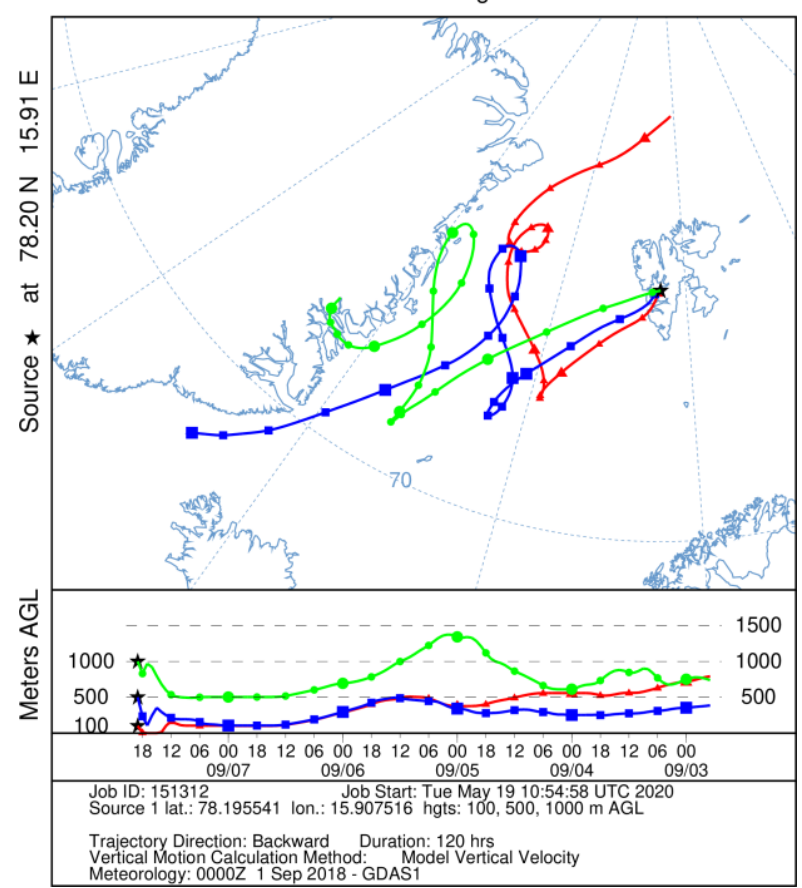

NOAA HYSPLIT MODEL

Backward trajectories ending at 1300 UTC 26 Sep 18 GDAS Meteorological Data

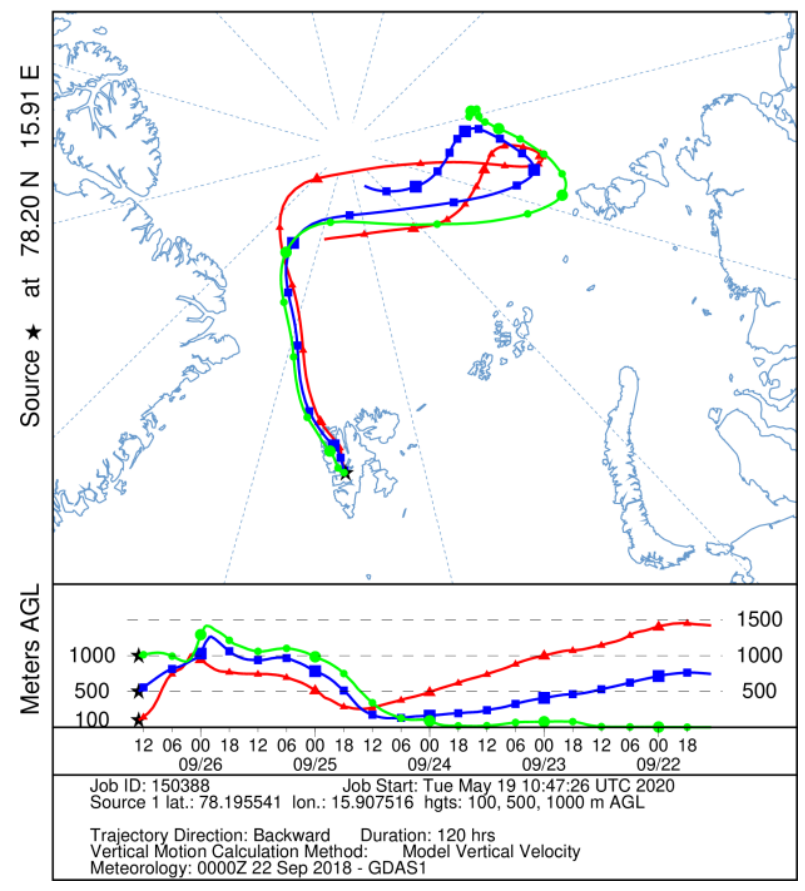

NOAA HYSPLIT MODEL

Backward trajectories ending at 1300 UTC 14 Sep 18 GDAS Meteorological Data

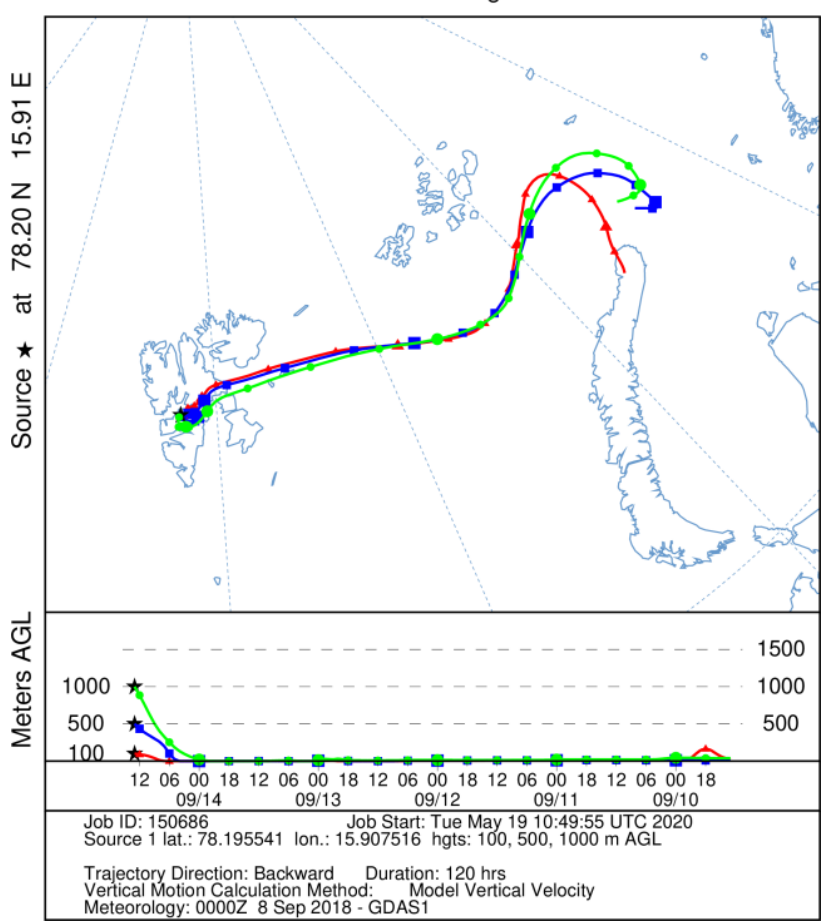

NOAA HYSPLIT MODEL

Backward trajectories ending at 1300 UTC 27 Sep 18 GDAS Meteorological Data

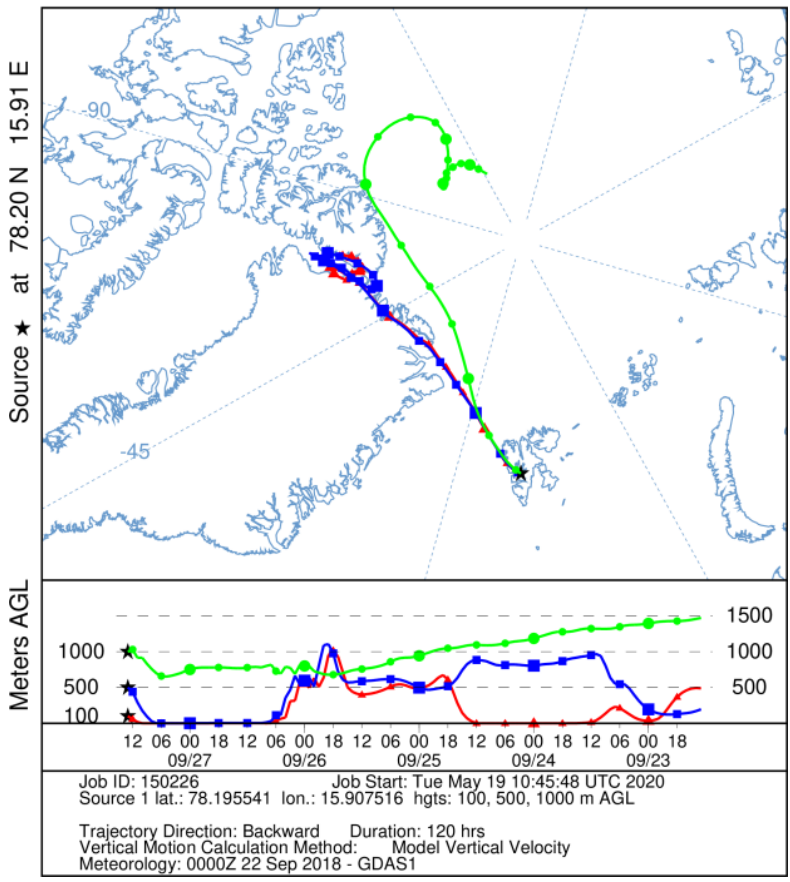


Figure S4 continued

NOAA HYSPLIT MODEL

Backward trajectories ending at 1800 UTC 28 Sep 18

GDAS Meteorological Data

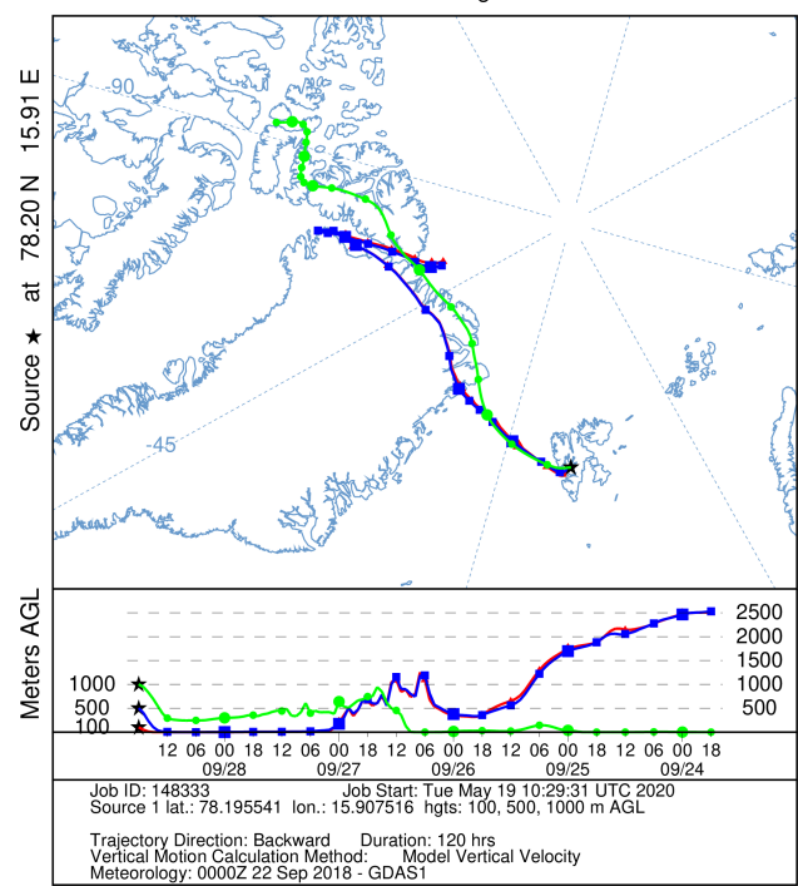

*calculated using the US NOAA Hybrid Single Particle Lagrangian Integrated Trajectory (HYSPLIT) model 

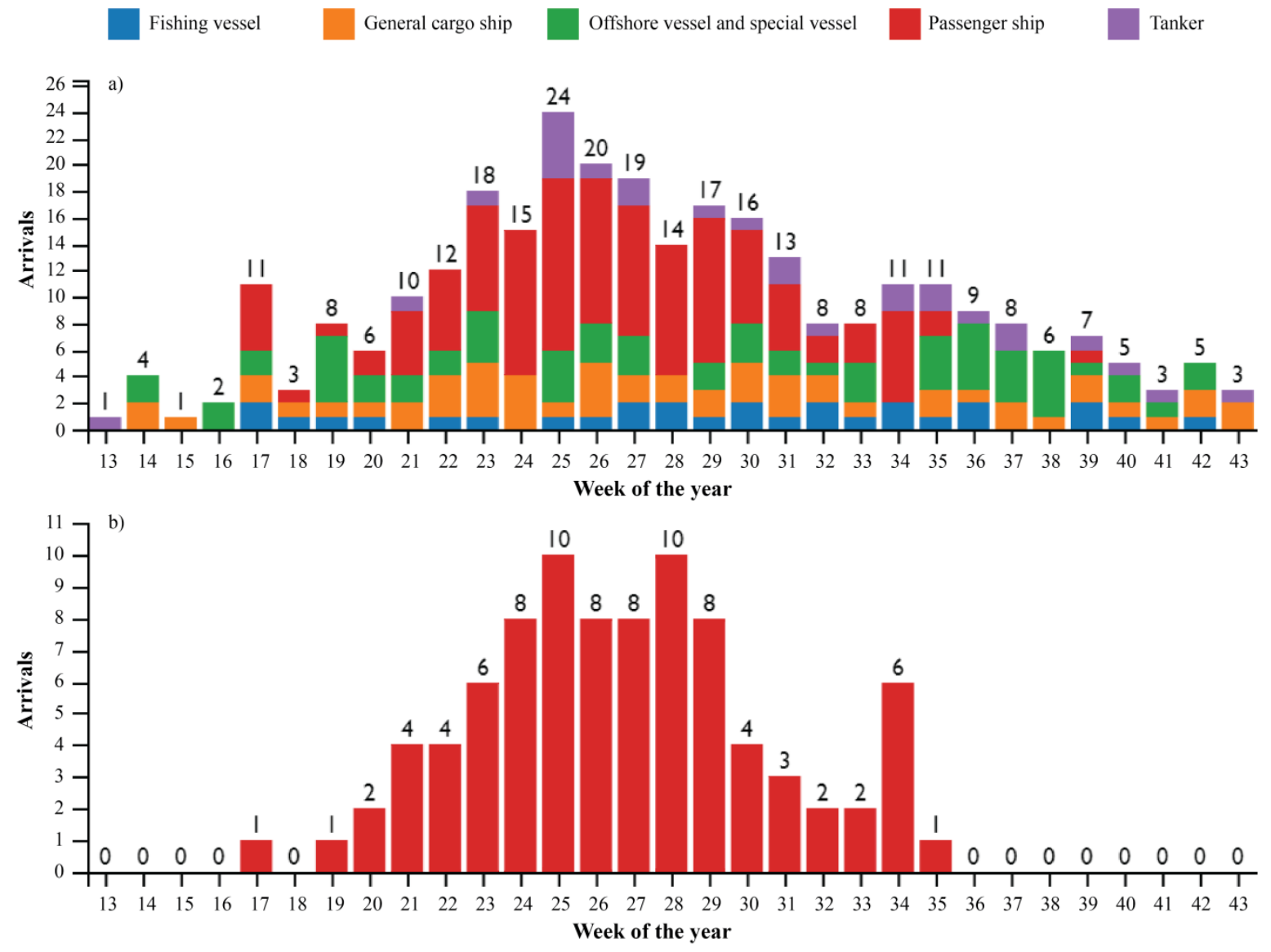

*data acquired from https://kystdatahuset.no/, last access: 30 May 2020

**this study sampling was performed during week $34-38$ period 


\section{References}

340 Alam, M. S., Delgado-Saborit, J. M., Stark, C., and Harrison, R. M.: Investigating PAH relative reactivity using congener profiles, quinone measurements and back trajectories, Atmos. Chem. Phys., 14, 2467-2477, https://doi.org/10.5194/acp-14-2467-2014, 2014.

Albinet, A., Leoz-Garziandia, E., Budzinski, H., and Villenave, E.: Simultaneous analysis of oxygenated and nitrated polycyclic aromatic hydrocarbons on standard reference material 1649a (urban dust) and on natural

345 ambient air samples by gas chromatography-mass spectrometry with negative ion chemical ionisation, J. Chromatogr. A, 1121, 106-113, https://doi.org/10.1016/j.chroma.2006.04.043, 2006.

Albinet, A., Leoz-Garziandia, E., Budzinski, H., and Villenave, E.: Polycyclic aromatic hydrocarbons (PAHs), nitrated PAHs and oxygenated PAHs in ambient air of the Marseilles area (South of France): Concentrations and sources, Science of the Total Environment, 384, 280-292, 10.1016/j.scitotenv.2007.04.028, 2007.

350 Albinet, A., Leoz-Garziandia, E., Budzinski, H., Villenave, E., and Jaffrezo, J. L.: Nitrated and oxygenated derivatives of polycyclic aromatic hydrocarbons in the ambient air of two French alpine valleys - Part 1 :

Concentrations, sources and gas/particle partitioning, Atmos. Environ., 42, 43-54, https://doi.org/10.1016/j.atmosenv.2007.10.009, 2008a.

Albinet, A., Leoz-Garziandia, E., Budzinski, H., Villenave, E., and Jaffrezo, J. L.: Nitrated and oxygenated 355 derivatives of polycyclic aromatic hydrocarbons in the ambient air of two French alpine valleys. Part 1: Concentrations, sources and gas/particle partitioning, Atmos. Environ., 42, 43-54, http://doi.org/10.1016/j.atmosenv.2007.10.009, 2008b.

Albinet, A., Tomaz, S., and Lestremau, F.: A really quick easy cheap effective rugged and safe (QuEChERS) extraction procedure for the analysis of particle-bound PAHs in ambient air and emission samples, Sci. Total

360 Environ., 450-451, 31-38, https://doi.org/10.1016/j.scitotenv.2013.01.068, 2013.

Albinet, A., Nalin, F., Tomaz, S., Beaumont, J., and Lestremau, F.: A simple QuEChERS-like extraction approach for molecular chemical characterization of organic aerosols: application to nitrated and oxygenated PAH derivatives (NPAH and OPAH) quantified by GC-NICIMS, Anal. Bioanal.Chem., 406, 3131-3148, https://doi.org/10.1007/s00216-014-7760-5, 2014.

365 Brorström-Lundén, E., Remberger, M., Kaj, L., Hansson, K., Palm Cousins, A., and Andersson, H.: Results from the Swedish national screening programme 2008, IVL Swedish Environmental Research Institute, Göteborg, Sweden, 69, 2010.

Burns, D. T., Danzer, K., and Townshend, A.: Use of the term" recovery" and" apparent recovery" in analytical procedures (IUPAC Recommendations 2002), Pure Appl. Chem., 74, 2201-2205,

370 https://doi.org/10.1351/pac200274112201., 2002.

Feilberg, A., Poulsen, M. W. B., Nielsen, T., and Skov, H.: Occurrence and sources of particulate nitropolycyclic aromatic hydrocarbons in ambient air in Denmark, Atmos. Environ., 35, 353-366, https://doi.org/10.1016/s1352-2310(00)00142-4, 2001.

Han, M., Kong, J., Yuan, J., He, H., Hu, J., Yang, S., Li, S., Zhang, L., and Sun, C.: Method development for 375 simultaneous analyses of polycyclic aromatic hydrocarbons and their nitro-, oxy-, hydroxy- derivatives in sediments, Talanta, 205, 120128, https://doi.org/10.1016/j.talanta.2019.120128, 2019.

Hsu, W. T., Liu, M. C., Hung, P. C., Chang, S. H., and Chang, M. B.: PAH emissions from coal combustion and waste incineration, J. Hazard. Mater., 318, 32-40, http://doi.org/10.1016/j.jhazmat.2016.06.038, 2016.

Huang, B., Liu, M., Bi, X., Chaemfa, C., Ren, Z., Wang, X., Sheng, G., and Fu, J.: Phase distribution, sources

380 and risk assessment of PAHs, NPAHs and OPAHs in a rural site of Pearl River Delta region, China, Atmos. Pollut. Res., 5, 210-218, https://doi.org/10.5094/APR.2014.026, 2014a.

Huang, B., Liu, M., Bi, X. H., Chaemfa, C., Ren, Z. F., Wang, X. M., Sheng, G. Y., and Fu, J. M.: Phase distribution, sources and risk assessment of PAHs, NPAHs and OPAHs in a rural site of Pearl River Delta region, China, Atmospheric Pollution Research, 5, 210-218, https://doi.org/10.5094/apr.2014.026, 2014 b.

385 Kanan, R., Andersson, J. T., Receveur, J., Guyomarch, J., Le Floch, S., and Budzinski, H.: Quantification of polycyclic aromatic compounds (PACs), and alkylated derivatives by gas chromatography-tandem mass spectrometry (GC/MS/MS) to qualify a reference oil, in: Proceedings of the Thirty-fifth AMOP technical seminar on environmental contamination and response, Ottawa, Canada, 2012, 616-927, 2012.

Konieczka, P., and Namieśnik, J.: Limit of detection and limit of quantification, in: Quality assurance and

390 quality control in the analytical chemical laboratory, CRC Press, Taylor \& Francis Group, Boca Raton, Florida, USA, 143-160, 2009.

Lammel, G., Kitanovski, Z., Kukucka, P., Novak, J., Arangio, A. M., Codling, G. P., Filippi, A., Hovorka, J., Kuta, J., Leoni, C., Pribylova, P., Prokes, R., Sanka, O., Shahpoury, P., Tong, H. J., and Wietzoreck, M.: Oxygenated and nitrated polycyclic aromatic hydrocarbons in ambient air-levels, phase partitioning, mass size distributions, and inhalation bioaccessibility, Environ. Sci. Technol., 54, 2615-2625,

https://doi.org/10.1021/acs.est.9b06820, 2020. 
Launiainen, J., and Vihma, T.: Derivation of turbulent surface fluxes - An iterative flux-profile method allowing arbitrary observing heights, Environmental Software, 5, 113-124, https://doi.org/10.1016/02669838(90)90021-W, 1990.

400 Li, J., Li, X., Li, M., Lu, S., Yan, J., Xie, W., liu, C., and Qi, Z.: Influence of air pollution control devices on the polycyclic aromatic hydrocarbon distribution in flue gas from an ultralow-emission coal-fired power plant, Energy \& Fuels, 30, 9572-9579, http://doi.org/10.1021/acs.energyfuels.6b01381, 2016.

Li, W., Wang, C., Shen, H. Z., Su, S., Shen, G. F., Huang, Y., Zhang, Y. Y., Chen, Y. C., Chen, H., Lin, N., Zhuo, S. J., Zhong, Q. R., Wang, X. L., Liu, J. F., Li, B. G., Liu, W. X., and Tao, S.: Concentrations and origins of nitro-polycyclic aromatic hydrocarbons and oxy-polycyclic aromatic hydrocarbons in ambient air in urban and rural areas in northern China, Environ. Pollut., 197, 156-164, https://doi.org/10.1016/j.envpol.2014.12.019, 2015 .

Meij, R., and Te Winkel, H.: The emissions of heavy metals and persistent organic pollutants from modern coalfired power stations, Atmos. Environ., 41, 9262-9272, https://doi.org/10.1016/j.atmosenv.2007.04.042, 2007.

410 Observation data of atmospheric PAHs at Zeppelin and Birkenes stations in 2018: http://ebas.nilu.no, access: 25 June 2020, 2018.

Rigamonti, L., Grosso, M., and Biganzoli, L.: Environmental assessment of refuse-derived fuel co-combustion in a coal-fired power plant, J. Ind. Ecol., 16, 748-760, http://doi.org/10.1111/j.1530-9290.2011.00428.x, 2012. Şengül, Ü.: Comparing determination methods of detection and quantification limits for aflatoxin analysis in 415 hazelnut, J. Food Drug Anal., 24, 56-62, https://doi.org/10.1016/j.jfda.2015.04.009, 2016.

Shrivastava, A., and Gupta, V. B.: Methods for the determination of limit of detection and limit of quantitation of the analytical methods, Chronicles of young scientists, 2, 21, https://doi.org/10.4103/2229-5186.79345, 2011. Tomaz, S., Shahpoury, P., Jaffrezo, J.-L., Lammel, G., Perraudin, E., Villenave, E., and Albinet, A.: One-year study of polycyclic aromatic compounds at an urban site in Grenoble (France): Seasonal variations, gas/particle partitioning and cancer risk estimation, Sci. Total Environ., 565, 1071-1083, http://doi.org/10.1016/j.scitotenv.2016.05.137, 2016.

Wang, R., Liu, G., and Zhang, J.: Variations of emission characterization of PAHs emitted from different utility boilers of coal-fired power plants and risk assessment related to atmospheric PAHs, Sci. Total Environ., 538, 180-190, http://doi.org/10.1016/j.scitotenv.2015.08.043, 2015.

425 Yu, Y., Katsoyiannis, A., Bohlin-Nizzetto, P., Brorstrom-Lunden, E., Ma, J. M., Zhao, Y., Wu, Z. Y., Tych, W., Mindham, D., Sverko, E., Barresi, E., Dryfhout-Clark, H., Fellin, P., and Hung, H.: Polycyclic Aromatic Hydrocarbons Not Declining in Arctic Air Despite Global Emission Reduction, Environ. Sci. Technol., 53, 2375-2382, 10.1021/acs.est.8b05353, 2019. 